\title{
4. \\ Über das Gleichgewicht und die Bewegung einer elastischen Scheibe.
}

(Von G. Kirchhoff; Privatdocent an der Universităt zu Berlin.)

Der erste Versuch einer Theorie der Transversalschwingungen elastischer Scheiben ist von Sophie Germain bekannt gemacht. Im Jahre 1811 reichte sie der Pariser Akademie, die einen Preis für eine solche Theorie ansgesetzt hatte, eine Abhandlung ein, in welcher eine Hypothese über die Kräfte auseinandergesetzt war, mit denen eine Scheibe Formveränderungen widerstrebt, und aus dieser Hypothese war eine partielle Differentialgleichung für die Schwingungen abgeleitet. Die Verfasserin hatte bei der Rechnung einen Fehler gemacht; Lagrange, der sich in der zur Begutachtung der Abhandlung niedergesetzten Commission befand, leitete aus ihrer Hypothese die Differentialgleichung ab, welche eine richtige Rechnung geben mufste. Es ist dieses dieselbe, welche noch jetzt als die richtige anerkannt wird. Noch fehlten aber die Grenzbedingungen, durch welche die Lösung der partiellen Differentialgleichung erst zu einer bestimmten wird. Diese hat Sophie Germain in einer zweiten Abhandlung, die sie 2 Jahre später der Akademie übergab, aus derselben Hypothese abgeleitet. Sie waren von der Art, dafs die Verfasserin die Lösung des Problems für den Fall rechteckiger Scheiben ermitteln konnte. Sie verglich ihre theoretischen Resultate für diesen Fall mit Beobachtungen und fand eine Übereinstimmung, die ihre Hypothese zu bestätigen schien. In einer dritten $\mathbf{A b}-$ handlung, die sie 1815 der Akademie überreichte, erweiterte sie ihre Hypothese so, dafs sich aus derselben auch die Theorie der Schwingungen von Platten ableiten liefs, die im natürlichen Zustande gekrümmt sind. Sie konnte die Rechnung für cylinderförmig gekrümmte Platten durchführen und fand auch hier ihre theoretischen Resultate im Einklang mit experimentellen.

Diese 3 Abhandlungen sind nicht gedruckt; den Hauptinhalt derselben und die Ergebnisse ihrer fortgesetzten Forschungen hat die Verfasserin in zwei Schriften veröffentlicht, von denen die erste „Recherches sur la théorie des surfaces élastìques" im Jahre 1821, die zweite „Remarques sur la nature, 
les bornes et l'étendue de la question des surfaces élastiques, et l'équation générale de ces surfaces" im Jahre 1826 zu Paris erschienen sind.

Ungeachtet der Bestätigungen, welche die Theorie von Sophie Germain durch Versuche erfahren hat, ist sie nicht richtig; denn man kann Folgerungen aus ihr ziehen, welche in offenbarem Widerspruche mit der Wirklichkeit stehen. Ich beschränke mich, um dieses zu zeigen, auf die Betrachtung einer Platte, die im natürlichen Zustande eben ist. Die Schlüsse, durch deren Hülfe Sophie Germain zu ihren Gesetzen für die Formveränderung, die eine solche Platte durch die Wirkung äufserer Kräfte erleidet, und für die Schwingungen, die sie vollführt, gelangt, sind im Wesentlichen folgende.

In jedem Elemente der Platte, welches seine Gestalt verändert hat, ist eine Kraft erzeugt, welche dasselbe in seine ursprüngliche Form zurückzuführen trachtet. Die Bedingung des Gleichgewichts der Platte ist die, dafs das Moment aller in derselben erzeugten Kräfte und das Moment der gegebenen äufseren Kräfte eine verschwindende Summe liefern. Es sei $\varepsilon$ die Dicke der Platte, $d f$ ein Element ihrer Mittelfläche; die in dem Elemente $\varepsilon d f$ erzeugte Kraft wird um so gröfser sein, je gröfser der Unterschied der Gestalt von $d f$ nach der Formveränderung und der ursprünglichen Gestalt dieses Elements ist. Hätte man ein passendes Maafs für diesen Unterschied, so würde man jene Kraft diesem proportional annehmen können; es sei $u$ ein solches Maafs, dann wird man jene Kraft

$$
=\boldsymbol{N}^{2} \boldsymbol{u} d f
$$

setzen können; wo $\mathrm{N}^{2}$ eine von der Dicke und der Natur der Platte abhängige Constante bezeichnet. Das Streben dieser Kraft geht dahin, $u$ zu verkleinern; das Moment derselben wird daher sein:

\section{$-\mathbf{N}^{2} u \delta \boldsymbol{u} d \boldsymbol{f}$}

wo $\delta u$ die virtuelle Veränderung von $u$ bedeutet.

Stellt man die entsprechende Betrachtung für den Fall eines elastischen Stabes an, so gelangt man zu den richtigen Endgleichungen, wenn man $u=$ dem reciproken Krümmungsradius der Mittellinie des Stabes setzt; Sophie Germain glaubte dem entsprechend in dem Falle einer Scheibe $u=$ der Summe der reciproken Hauptkrümmungsradien der Mittelfläche annehmen zu können. Sind diese Hauptkrümmungstradien $=\varrho_{1}$ und $\varrho_{2}$, so erhielt sie demnach für das Moment der in dem einen Elemente erzeugten Kraft den Ausdruck

$$
-N^{2}\left(\frac{1}{\varphi_{1}}+\frac{1}{\rho_{2}}\right) \delta\left(\frac{1}{\varrho_{1}}+\frac{1}{\varrho_{2}}\right) d f
$$


und als Bedingung des Gleichgewichts der Platte die Gleichung:

$$
\delta \boldsymbol{P}-\boldsymbol{N}^{2} \int\left(\frac{1}{\varrho_{1}}+\frac{1}{\varrho_{2}}\right) \delta\left(\frac{1}{\varrho_{1}}+\frac{1}{\varrho_{2}}\right) d f=0,
$$

falls $\delta \boldsymbol{P}$ das Moment der gegebenen äufsern Kräfte bezeichnet.

Um zu zeigen, dafs diese Bedingung unmöglich die richtige sein kann, wende ich sie auf den Fall an, wo eine Scheibe unendlich wenig aus ihrer ursprünglichen Gestalt gebracht ist, durch Kräfte, die auf ihr Inneres senkrecht zu ihrer Mittelfläche wirken; den Rand der Scheibe nehme ich dabei der Einfachheit wegen als frei an. Die Mittelfläche in ihrer ursprünglichen Gestalt sei die $x y$ Ebene eines rechtwinkligen Coordinatensystems, $z$ die auf ihr senkrechte Verrückung, welche der Punct $(x, y)$ der Mittelfläche erlitten hat, $\boldsymbol{Z}$ die Kraft, die in der Richtung von $z$ auf eine Linie der Platte wirkt, die in derselben Richtung durch den Punct $(x, y)$ gezogen ist. Setzt man dann

$$
\frac{\partial^{2} z}{\partial x^{2}}+\frac{\partial^{2} z}{\partial y^{2}}=u,
$$

so liefert jene Gleichgewichtsbedingung für $u$ die partielle Differentialgleichung

$$
\mathbf{N}^{2}\left(\frac{\partial^{2} u}{\partial x^{2}}+\frac{\partial^{2} u}{\partial y^{2}}\right)=\mathbf{Z}
$$

und die Grenzbedingungen

$$
\boldsymbol{u}=\mathbf{0}, \quad \frac{\partial u}{\partial \boldsymbol{n}}=\mathbf{0}
$$

wo $n$ die Normale der Contour der Mittelfläche bezeichnet. Nun ist aber die Lösung der Differentialgleichung für $u$ schon durch die erste der beiden Grenzbedingungen vollkommen bestimmt; es ist daher im Allgemeinen nicht möglich, ein $u$ zu finden, welches auch noch der zweiten Grenzbedingung genügt; und demnach gäbe es im Allgemeinen gar kein Gleichgewicht für die Platte. Wären die gegebenen Kräfte $\boldsymbol{Z}$ von der Art, dafs eine Function $\boldsymbol{u}$ gefunden werden könnte, die den beiden Grenzbedingungen genügt, so hätte man, um die Gestalt der Mittelfläche zu ermitteln, diesen Werth von $u$ in die Differentialgleichung für $z \mathrm{zu}$ substituiren und aus derselben $z$ zu bestimmen. Dieser Gleichung genügen aber unendlich viele Functionen; es würde daher in diesem Falle unendlich viele Gleichgewichtslagen der Platte geben. Dieser Fall würde z. B. eintreten, wenn keine Kräfte $\boldsymbol{Z}$ vorhanden sind; wäre die Platte in irgend welche Gestalt gebracht, für die

$$
\frac{\partial^{2} z}{\partial x^{2}}+\frac{\partial^{2} z}{\partial y^{2}}=0
$$

ist, und dann sich selbst überlassen, so müfste sie in dieser Gestalt verharren, 
ohne das Bestreben zu zeigen, in ihre ursprüngliche Gestalt zurückzukehren. Jener Gleichgewichtsbedingung zufolge müfste die Platte, auch wenn sie endliche Krümmungen erlitten hat, ohne Mitwirkung äufserer Kräfte im Gleichgewichte sich befinden, sobald für alle Puncte ihrer Mittelfläche die Summe der reciproken Hauptkrümmungsradien verschwindet.

Eine zweite Theorie des Gleichgewichts und der Bewegung elastischer Scheiben ist von Poisson aufgestellt und in seiner berühmten Abhandlung „Sur l'équilibre et le mouvement des corps élastiques" *) entwickelt. Aber auch diese Theorie bedarf einer Berichtigung, und dieselbe zu geben, ist eben meine Absicht. Poisson gelangt, indem er seine allgemeinen Gleichungen des Gleichgewichts elastischer Körper auf den Fall einer Scheibe anwendet, zu derselben partiellen Differentialgleichung, zu welcher die Hypothese von Sophie Germain geführt hat, aber zu andern Grenzbedingungen, und zwar zu drei Grenzbedingungen. Ich werde beweisen, dafs im Allgemeinen diesen nicht gleichzeitig genügt werden kann; woraus dann folgt, dafs auch nach der Poissonschen Theorie eine Platte im Allgemeinen keine Gleichgewichtslage haben müfste. Diesen Beweis werde ich aber erst führen, nachdem ich die zwei Grenzbedingungen abgeleitet haben werde, die an die Stelle der Poissonschen drei zu setzen sind, weil er sich naturgemäfs an die Betrachtungen anschliefst, durch welche ich jene ableiten will.

Poisson hat seine Theorie auf den Fall einer kreisförmigen Platte angewandt, die so schwingt, dafs alle Puncte, die gleich weit von ihrem Mittelpuncte abstehen, sich immer in demselben Zustande befinden; er konnte sie auf diesen Fall anwenden, weil in demselben eine seiner drei Grenzbedingungen identisch erfüllt wurde. Aus der modificirten Theorie werde ich allgemein die Gesetze der Schwingungen einer freien kreisförmigen Platte entwickeln; in dem bezeichneten speciellen Falle werde ich zu denselben Formeln gelangen, welche Poisson gefunden hat. Durch die Güte des Herrn Director Strehlke, welcher Messungen in Bezug auf die Knotenlinien kreisförmiger Scheiben angestellt hat, bin ich in den Stand gesetzt, einige der numerischen Resultate der Theorie mit den entsprechenden Resultaten der Beobachtung zusammenzustellen.

\$. 1.

Poisson legt seinen Betrachtungen über eine elastische Platte die Gleichungen zu Grunde, welche auf die Formveränderungen beliebig gestalteter

*) Mém. de l'Ac. d. sc. à Par. 1829. p. 357. 
elastischer Körper sich beziehen. Diese Gleichungen lassen sich in eine zusammenfassen, welche ausspricht, dafs das Moment der Kräfte, welche die Formveränderung bewirkt haben, der Variation eines gewissen Integrals gleich ist. Es hat diese vor jenen auch das voraus, dafs jene nur gelten, wenn die Verrückungen unendlich klein sind, während diese besteht, sobald die Dilatationen und Contractionen unendlich klein sind; auf den Fall von unendlich dünnen Stäben oder Platten, die endliche Krümmungen erlitten haben, können jene nicht angewandt werden, diese kann es aber. Sie ist die folgende:

$$
\text { (1.) } \quad 0=\delta \boldsymbol{P}-\delta \boldsymbol{K} \int \boldsymbol{d} \boldsymbol{V}\left(\lambda_{1}^{2}+\lambda_{2}^{2}+\lambda_{3}^{2}+\theta\left(\lambda_{1}+\lambda_{2}+\lambda_{3}\right)^{2}\right) \text {. }
$$

Hier bedeuten $\delta \boldsymbol{P}$ das Moment der gegebenen Kräfte, $\boldsymbol{d} \boldsymbol{V}$ das Volumen eines Elements des Körpers, $\lambda_{1}, \lambda_{2}, \lambda_{3}$ die Hauptdilatationen dieses Elements; die Integration ist über den ganzen Körper auszudehnen; $K$ und $\theta$ sind zwei Constanten, von denen der Elasticitätscoëfficient $q$ auf die Weise abhängt, dafs

$$
q=2 K \frac{1+3 \theta}{1+2 \theta}
$$

ist. Man erhält aus (1.) die Poissonschen Gleichungen, wenn man $\theta=\frac{1}{2}$ setzt, und die Gleichungen, zu welchen Herr Werthein durch seine Versuche geführt worden ist ${ }^{*}$ ), wenn man $\theta=1$ macht. Bezeichnet man die rechtwinkligen Coordinaten des Ortes von $\boldsymbol{d V}$ im ursprünglichen Zustande des Körpers durch $x, y, z$, die Verrückungen in den Richtungen der Axen, die dasselbe bei der Formveränderung erlitten hat, durch $u, v, w$, die Kräfte, die auf dasselbe in den Richtungen der Axen wirken, durch $\boldsymbol{X} d \boldsymbol{V}, \boldsymbol{Y} d \boldsymbol{V}, \boldsymbol{Z} d \boldsymbol{V}$; nennt man ferner $d 0$ ein Element der Oberfläche des Körpers und $(\boldsymbol{X}) d 0,(\boldsymbol{Y}) d 0,(\boldsymbol{Z}) d 0$ die Druckkräfte, die auf dasselbe in den Richtungen der Axen ausgeübt werden, so ist der Werth von $\delta \boldsymbol{P}$, der in die Gleichung (1.) gesetzt werden mufs, folgender :

(2.) $\left.\delta \boldsymbol{P}=\int d \boldsymbol{V}(\boldsymbol{X} \delta \boldsymbol{u}+\boldsymbol{Y} \delta \boldsymbol{v}+\boldsymbol{Z} \delta \boldsymbol{w})+\int d 0((\boldsymbol{X}) \delta \boldsymbol{u}+(\boldsymbol{Y}) \delta \boldsymbol{v}+(\boldsymbol{Z}) \delta \boldsymbol{w})\right)$; wo das erste Integral über das ganze Volumen, das zweite über die ganze Oberfläche des Körpers auszudehnen ist.

Dafs die Gleichung (1.) für den Fall, dafs $u, v, v$ unendlich klein sind, wirklich die bekannten Gleichungen für die Formveränderungen elastischer Körper liefert, davon überzeugt man sich leicht durch folgende Rechnung.

Es seien $\alpha, \beta, \gamma$ die Cosinus der Winkel, welche eine durch den Punct $(x, y, z)$ gezogene Linie mit den Coordinaten-Axen bildet: die Dilatation

*) Ann. de ch. et de ph. $3^{e}$ sér. XXIII. p. 52. 
in der Richtung dieser Linie für den Punct $(x, y, z)$, die durch $\lambda$ bezeichnet werden möge, ist dann unter der Voraussetzung, dafs $u, v, w$, also auch die Differentialquotienten dieser Gröfsen nach $x, y, z$, unendlich klein sind, durch die folgende Gleichung bestimmt:

$$
\begin{aligned}
\lambda=\alpha^{2} \frac{\partial u}{\partial x} & +\beta^{2} \frac{\partial v}{\partial y}+\gamma^{2} \frac{\partial w}{\partial z} \\
& +\beta \gamma\left(\frac{\partial w}{\partial y}+\frac{\partial v}{\partial z}\right)+\gamma \alpha\left(\frac{\partial u}{\partial z}+\frac{\partial w}{\partial x}\right)+\alpha \beta\left(\frac{\partial v}{\partial x}+\frac{\partial u}{\partial y}\right) .
\end{aligned}
$$

Die Hauptdilatationen $\lambda_{1}, \lambda_{2}, \lambda_{3}$ sind die Werthe von $\lambda$. für diejenigen Werthe von $\alpha, \beta, \gamma$, für welche die Variation $\delta 2$ verschwindet; d. h. sie sind die Wurzeln der Gleichung

$$
\begin{aligned}
\mathbf{0}= & \left(\frac{\partial u}{\partial x}-\lambda\right)\left(\frac{\partial v}{\partial y}-\lambda\right)\left(\frac{\partial w}{\partial z}-\lambda\right) \\
& -\frac{1}{4}\left(\frac{\partial u}{\partial x}-\lambda\right)\left(\frac{\partial w}{\partial y}+\frac{\partial v}{\partial z}\right)^{2}-\frac{1}{4}\left(\frac{\partial v}{\partial y}-\lambda\right)\left(\frac{\partial u}{\partial z}+\frac{\partial w}{\partial x}\right)^{2}-\frac{1}{4}\left(\frac{\partial w}{\partial z}-\lambda\right)\left(\frac{\partial v}{\partial x}+\frac{\partial u}{\partial y}\right)^{2} \\
& +\frac{1}{4}\left(\frac{\partial w}{\partial y}+\frac{\partial v}{\partial z}\right)\left(\frac{\partial u}{\partial z}+\frac{\partial w}{\partial x}\right)\left(\frac{\partial v}{\partial x}+\frac{\partial u}{\partial y}\right) .
\end{aligned}
$$

Es folgt daraus :

und ferner

$$
\lambda_{1}+\lambda_{2}+\lambda_{3}=\frac{\partial u}{\partial x}+\frac{\partial v}{\partial y}+\frac{\partial w}{\partial z}
$$

$$
\begin{aligned}
\lambda_{2} \lambda_{3}+\lambda_{3} \lambda_{1}+\lambda_{1} \lambda_{2}= & \frac{\partial v}{\partial y} \frac{\partial w}{\partial z}+\frac{\partial w}{\partial z} \frac{\partial u}{\partial x}+\frac{\partial u}{\partial x} \frac{\partial v}{\partial y} \\
& -\frac{1}{4}\left(\frac{\partial w}{\partial y}+\frac{\partial v}{\partial z}\right)^{2}-\frac{1}{4}\left(\frac{\partial u}{\partial z}+\frac{\partial w}{\partial x}\right)^{2}-\frac{1}{4}\left(\frac{\partial v}{\partial x}+\frac{\partial u}{\partial y}\right)^{2}
\end{aligned}
$$

also auch

$$
\begin{aligned}
\lambda_{1}^{2}+\lambda_{2}^{2}+\lambda_{3}^{2}= & \left(\frac{\partial u}{\partial x}\right)^{2}+\left(\frac{\partial v}{\partial y}\right)^{2}+\left(\frac{\partial w}{\partial z}\right)^{2} \\
& +\frac{1}{2}\left(\frac{\partial w}{\partial y}+\frac{\partial v}{\partial z}\right)^{2}+\frac{1}{2}\left(\frac{\partial u}{\partial z}+\frac{\partial w}{\partial x}\right)^{2}+\frac{1}{2}\left(\frac{\partial v}{\partial x}+\frac{\partial u}{\partial y}\right)^{2} .
\end{aligned}
$$

Diese Werthe von $\lambda_{1}+\lambda_{2}+\lambda_{3}$ und $\lambda_{1}^{2}+\lambda_{2}^{2}+\lambda_{3}^{2}$ sind in die Gleichung (1.) zu substituiren *). Wir schreiben dieselbe wie folgt:

$$
\delta \boldsymbol{P}-\boldsymbol{K} \delta \Omega=0
$$

*) Die Gleíchung, die durch diese Substitution 'entsteht, findet sich in wenig veränderter Gestalt schon in einer Abhandlung von G. Green, $O n$ the laws of reflexion and refraction of light, Camb. Phil. Trans. VII."; sie ist dort auf andere Weise, als hier, ohne Betrachtung der Hauptdilatationen abgeleitet. 
4. Kirchhoff, üb. d. Gleichgewicht u. d. Beweyung einer elastischen Scheibe. 57

indem wir

$$
\text { (4.) } \Omega=\int d V\left(\lambda_{1}^{2}+\lambda_{2}^{2}+\lambda_{3}^{2}+\theta\left(\lambda_{1}+\lambda_{2}+\lambda_{3}\right)^{2}\right)
$$

setzen. Die Variation $\delta \Omega$ wird aus drei Theilen bestehen, von denen der erste von $\delta u$, der zweite von $\delta v$, der dritte von $\delta w$ abhangt; diese drei Theile wollen wir durch $\delta \boldsymbol{R}, \delta \boldsymbol{S}, \delta \boldsymbol{T}$ bezeichnen; dann ergiebt sich

$$
\text { (5.) } \delta \Omega=\delta \boldsymbol{R}+\delta \boldsymbol{S}+\delta \boldsymbol{T} \text {, }
$$

und man findet:

$$
\begin{aligned}
\delta \boldsymbol{R}=\int \boldsymbol{d}\left\{\left(\boldsymbol{2}(1+\theta) \frac{\partial u}{\partial x}+2 \theta\right.\right. & \left.\frac{\partial v}{\partial y}+2 \theta \frac{\partial w}{\partial z}\right) \frac{\partial \delta u}{\partial x} \\
& \left.+\left(\frac{\partial v}{\partial x}+\frac{\partial u}{\partial y}\right) \frac{\partial \delta u}{\partial y}+\left(\frac{\partial w}{\partial x}+\frac{\partial u}{\partial z}\right) \frac{\partial \delta u}{\partial z}\right\} .
\end{aligned}
$$

Aus dem Ausdrucke von $\delta \boldsymbol{R}$ ergiebt sich der von $\delta \boldsymbol{S}$, und aus diesem der von $\delta \boldsymbol{T}$, wenn man $u, v, w$ und gleichzeitig $x, y, z$ cyklisch vertauscht. Den Ausdruck von $\delta \boldsymbol{R}$ zerlege man in drei Integrale, von denen das erste unter dem Integralzeichen den Factor $\frac{\partial \delta u}{\partial x}$, das zweite den Factor $\frac{\partial \delta u}{\partial y}$, das dritte den Factor $\frac{\partial \delta u}{\partial z}$ hat. Auf das erste von den dreien wende man den Satz an, der durch die Gleichung

$$
\int \boldsymbol{d V F} \frac{\partial \boldsymbol{G}}{\partial \boldsymbol{x}}=-\int \boldsymbol{d} \boldsymbol{V} \boldsymbol{G} \frac{\partial \boldsymbol{F}}{\partial x}-\int d \boldsymbol{d O F} \boldsymbol{G} \cos (\boldsymbol{N}, x)
$$

ausgesprochen wird, in welcher $\boldsymbol{F}$ und $\boldsymbol{G}$ zwei beliebige Functionen von $x, y, z$ bezeichnen, $\boldsymbol{d} \boldsymbol{V}$ das Element eines begrenzten Raums, $\boldsymbol{d O}$ das Element der Oberfläche desselben und $(\boldsymbol{N}, \boldsymbol{x})$ den Winkel bedeutet, den die nach dem Innern des begrenzten Raumes gerichtete Normale von $d O$ mit der $x$ Axe bildet; auf das zweite und dritte der Integrale, deren Summe $\delta \boldsymbol{R}$ ist, wende man die Sätze an, die aus jenem durch Vertauschung von $x$ mit $y$ oder $z$ entstehen; jedesmal mache man dabei $G=\delta u$. Fassen wir dann die nach $d V$ zu nehmenden Integrale zusammen, und eben so die nach $d O$ zu nehmenden, so ergiebt sich

$$
\begin{aligned}
\delta \boldsymbol{R}= & -\int d \boldsymbol{V}\left\{\boldsymbol{2}(1+\theta) \frac{\partial^{2} u}{\partial x^{2}}+\frac{\partial^{2} u}{\partial y^{2}}+\frac{\partial^{2} u}{\partial z^{2}}+(1+2 \theta) \frac{\partial^{2} v}{\partial x \partial y}+(1+2 \theta) \frac{\partial^{2} w}{\partial x \partial z}\right\} \delta u \\
& -\int d \boldsymbol{O}\left\{\left(2(1+\theta) \frac{\partial u}{\delta x}+2 \theta \frac{\partial v}{\partial y}+2 \theta \frac{\partial w}{\partial z}\right) \cos (\boldsymbol{N}, x)\right. \\
& \left.+\left(\frac{\partial v}{\partial x}+\frac{\partial u}{\partial y}\right) \cos (\boldsymbol{N}, \boldsymbol{y})+\left(\frac{\partial w}{\partial x}+\frac{\partial u}{\partial z}\right) \cos (\boldsymbol{N}, \boldsymbol{z})\right\} \delta u .
\end{aligned}
$$

Bildet man aus diesem Ausdrucke von $\delta \boldsymbol{R}$ durch die angegebenen VertauCrelle's Journal f. d. M. Bd. XL. Heft 1. 
schungen die Ausdrücke von $\delta \boldsymbol{S}$ und $\delta \boldsymbol{T}$, und dann der Gleichung (5.) gemäfs den Werth von $\delta \Omega$; setzt diesen, so wie den Werth von $\delta \boldsymbol{P}$ aus (2.), in die Gleichung (3.), zieht hier die Integrale zusammen, die über das Volumen des Körpers auszudehnen sind, so wie diejenigen, die sich auf die Oberfläche desselben beziehen, und setzt dann, den Principien der Variationsrechnung gemäfs, die Factoren von $\delta u, \delta v, \delta w$ unter den beiden Integralzeichen $=0$; so erhält man folgende Gleichungen:

$$
\begin{aligned}
& \text { Für einen Punct im Innern des Körpers : } \\
& \boldsymbol{K}\left\{\boldsymbol{2}(1+\theta) \frac{\partial^{2} u}{\partial x^{2}}+\frac{\partial^{2} u}{\partial y^{2}}+\frac{\partial^{2} u}{\partial z^{2}}+(1+2 \theta) \frac{\partial^{2} v}{\partial x \partial y}+(1+2 \theta) \frac{\partial^{2} w}{\partial x \partial z}\right\}+\boldsymbol{X} \\
& =0 \text {, } \\
& \boldsymbol{K}\left\{\boldsymbol{2}(1+\theta) \frac{\partial^{2} v}{\partial y^{2}}+\frac{\partial^{2} v}{\partial z^{2}}+\frac{\partial^{2} v}{\partial x^{2}}+(1+2 \theta) \frac{\partial^{2} w}{\partial y \partial z}+(1+2 \theta) \frac{\partial^{2} u}{\partial y \partial x}\right\}+\boldsymbol{Y} \\
& =0 \text {, } \\
& \boldsymbol{K}\left\{\boldsymbol{2}(1+\theta) \frac{\partial^{2} w}{\partial z^{2}}+\frac{\partial^{2} w}{\partial x^{2}}+\frac{\partial^{2} w}{\partial y^{2}}+(1+2 \theta) \frac{\partial^{2} u}{\partial z \partial x}+(1+2 \theta) \frac{\partial^{2} v}{\partial z \partial y}\right\}+\mathbf{Z} \\
& =0 \text {. } \\
& \boldsymbol{K}\left\{\left(\boldsymbol{2}(1+\boldsymbol{\theta}) \frac{\partial u}{\partial x}+\boldsymbol{2} \boldsymbol{\theta} \frac{\partial v}{\partial y}+\boldsymbol{2} \boldsymbol{\theta} \frac{\partial w}{\partial z}\right) \cos (\boldsymbol{N}, x)\right. \\
& \left.+\left(\frac{\partial v}{\partial x}+\frac{\partial u}{\partial y}\right) \cos (\boldsymbol{N}, y)+\left(\frac{\partial w}{\partial x}+\frac{\partial u}{\partial z}\right) \cos (\boldsymbol{N}, \boldsymbol{z})\right\}+(\boldsymbol{X})=0 \\
& K\left\{\left(2(1+\theta) \frac{\partial v}{\partial y}+2 \theta \frac{\partial w}{\partial z}+2 \theta \frac{\partial u}{\partial x}\right) \cos (N, y)\right. \\
& \left.+\left(\frac{\partial w}{\partial y}+\frac{\partial v}{\partial z}\right) \cos (\boldsymbol{N}, \boldsymbol{z})+\left(\frac{\partial u}{\partial y}+\frac{\partial v}{\partial x}\right) \cos (\boldsymbol{N}, \boldsymbol{x})\right\}+(\boldsymbol{Y})=0 \\
& \boldsymbol{K}\left\{\left(\boldsymbol{2}(1+\theta) \frac{\partial w}{\partial z}+2 \theta \frac{\partial u}{\partial x}+2 \theta \frac{\partial v}{\partial y}\right) \cos (\mathbf{N}, \mathfrak{z})\right. \\
& \left.+\left(\frac{\partial u}{\partial z}+\frac{\partial w}{\partial x}\right) \cos (\boldsymbol{N}, \boldsymbol{x})+\left(\frac{\partial v}{\partial z}+\frac{\partial w}{\partial y}\right) \cos (\boldsymbol{N}, y)\right\}+(\boldsymbol{Z})=0 .
\end{aligned}
$$

Diese Gleichungen sind dieselben wie die, welche Caucley auf einem Wege abgeleitet hat, bei welchem er nicht auf die Betrachtung der Molecularkräfte zurückging; sie gehen in die Poisson'schen über, wenn man $\theta=\frac{1}{2}$ setzt, und in die Wertheim'schen, wenn $\theta=1$ gesetzt wird.

Ich werde jetzt eine Ableitung der Gleichung (1.) geben, aus welcher hervorgehen wird, dafs sie eine allgemeinere Gültigkeit hat, als die Gleichungen (6.). Betrachtungen, die denen welche hier folgen ganz ähnlich sind, hat 
Lagrange mehrmals in seiner Mechanik, z. B. bei der Herleitung der Gleichgewichtsbedingung eines elastischen Stabes angestellt.

Es sei $d V$ das Volumen eines unendlich kleinen Theils des elastischen Körpers in seinem natürlichen Zustande. Der Zustand, in welchen dieser Theil durch die Formveränderung geräth, kann bekanntlich aus dem natürlichen als auf die Weise hervorgegangen angesehen werden, dafs der Theil ohne Veränderung der relativen Lage seiner Molecülen eine andere Lage im Raume erhalten hat und dann in drei auf einander senkrechten Richtungen (gleichmäfsig in jeder, aber verschieden in den verschiedenen) dilatirt worden ist ${ }^{*}$ ). Eine unendlich kleine Kugel gebt hiernach in ein Ellipsoïd über, dessen Axen die Richtungen haben, in denen die Dilatationen Statt fanden. Diese Dilatationen werden daher die Hauptdilatationen $\lambda_{1}, \lambda_{2}, \lambda_{3}$ sein. Die Elasticität des Körpers bewirkt, dafs der betrachtete Theil sich in den Richtungen, in denen er ausgedehnt ist, zusammenzuziehen strebt; die Kräfte, mit denen er sich in denselben zusammenzuziehen strebt, seien $L_{1} d V, L_{2} d V, L_{3} d V$; die erste von ihnen sucht $\lambda_{1}$, die zweite $\lambda_{2}$, die dritte $\lambda_{3}$ zu verkleinern. Das Moment der ersten Kraft ist daher $-\boldsymbol{L}_{1} d \boldsymbol{V} \delta \lambda_{1}$, das der zweiten $-\boldsymbol{L}_{2} d \boldsymbol{V} \delta \lambda_{2}$, das der dritten $-\boldsymbol{L}_{3} d V \delta \lambda_{3}$, und das Gesammtmoment der drei Kräfte ist

$$
-d V\left(L_{1} \delta \lambda_{1}+L_{2} \delta \lambda_{2}+L_{3} \delta \lambda_{3}\right) .
$$

Nun sind $\boldsymbol{L}_{1}, \boldsymbol{L}_{2}, \boldsymbol{L}_{3}$ Functionen von $\lambda_{1}, \lambda_{2}, \lambda_{3}$. Wir wissen von ihnen, dafs sie gleichzeitig mit $\lambda_{1}, \lambda_{2}, \lambda_{3}$ verschwinden; ferner, dafs $L_{1}$ eine symmetrische Function von $\lambda_{2}$ und $\lambda_{3}$, und dieselbe Function von $\lambda_{1}, \lambda_{2}, \lambda_{3}$ wie $L_{2}$ von $\lambda_{2}, \lambda_{3}, \lambda_{1}$ und $L_{3}$ von $\lambda_{3}, \lambda_{1}, \lambda_{2}$ sein mufs. Nimmt man daher $\lambda_{1}, \lambda_{2}, \lambda_{3}$ als unendlich klein an, so wird man

$$
\begin{aligned}
& \boldsymbol{L}_{1}=\boldsymbol{a} \lambda_{1}+\boldsymbol{b} \lambda_{2}+\boldsymbol{b} \lambda_{3}, \\
& \boldsymbol{L}_{2}=\boldsymbol{b} \lambda_{1}+\boldsymbol{a} \lambda_{2}+\boldsymbol{b} \lambda_{3}, \\
& \boldsymbol{L}_{3}=\boldsymbol{b} \lambda_{1}+\boldsymbol{b} \lambda_{2}+\boldsymbol{a} \lambda_{3}
\end{aligned}
$$

setzen können; wo $a$ und $b$ zwei von der Natur des Körpers abhängige Gröfsen bezeichnen. Fübrt man an Stelle derselben zwei andere $K$ und $\theta$ ein, die mit ihnen durch die Gleichungen

$$
a=2 K(1+\theta), b=2 K \theta
$$

verbunden sind, so erhält man für das Moment der in $d V$ erzeugten Kräfte den Ausdruck

$$
-d V . \delta K\left(\lambda_{1}^{2}+\lambda_{2}^{2}+\lambda_{3}^{2}+\theta\left(\lambda_{1}+\lambda_{2}+\lambda_{3}\right)^{2}\right)
$$

*) Eine Compression will ich als negative Dilatation bezeichnen. 
und für das Moment aller in dem Körper erzeugten Kräfte den Ausdruck

$$
-\delta \boldsymbol{K} \int \boldsymbol{d} \boldsymbol{V}\left(\lambda_{1}^{2}+\lambda_{2}^{2}+\lambda_{3}^{2}+\theta\left(\lambda_{1}+\lambda_{2}+\lambda_{3}\right)^{2}\right) \text {. }
$$

Die Summe dieses Moments und des Moments der äufseren Kräfte mufs für den Gleichgewichtszustand verschwinden; wie es durch die Gleichung (1.) ausgesprochen wird.

\section{\$. 2.}

Jetzt wollen wir zur Betrachtung einer Platte übergehen. Wir setzen in Bezug auf dieselbe voraus, dafs ihre Grundflächen im natürlichen Zustande durch zwei parallele, unendlich nahe Ebenen gebildet werden, ihr Rand durch eine beliebige Cylinder-Oberfläche, die jene senkrecht schneidet. Die Platte hat eine Form-Änderung erlitten durch Kräfte, die auf ihr Inneres wirken und durch Druckkräfte, die auf ihren Rand ausgeübt werden, während ihre Grundflächen frei sind. Diese Kräfte sind endlich nur so grofs, dafs die Dilatationen, die sie hervorbringen, als unendlich klein betrachtet werden dürfen. Hierdurch ist jedoch nicht ausgesprochen, dafs die Krümmungen, die die Platte erlitten hat, unendlich klein sind; diese wollen wir uns vorläufig als endlich vorstellen.

Um die Anwendung der Gleichung (1.) auf den Fall einer solchen Platte zu vermitteln, machen wir zwei Annahmen, die wir als Ergebnisse des Experiments ansehen und die ganz entsprechend denjenigen sind, welche Jacob Bernoulli in Bezug auf einen elastischen Stab macht; nämlich folgende:

1) Jede gerade Linie der Platte, welche ursprünglich senkrecht auf den Grundflächen war, bleibt bei der Form-Änderung gerade und senkrecht auf den Flächen, welche ursprünglich den Grundflächen parallele Ebenen waren;

2) Alle Elemente der Mittelfläche (d. h. derjenigen Fläche, welche im natürlichen Zustande der Platte die Ebene ist, die den Grundflächen parallel in der Mitte zwischen diesen liegt) erleiden bei der Form-Änderung keine Dilatation.

Mit Hülfe dieser beiden Annahmen werden sich die Werthe der Hauptdilatationen $\lambda_{1}, \lambda_{2}, \lambda_{3}$ für den gegenwärtigen Fall leicht ausdrücken lassen durch die Hauptkrümmungsradien der Mittelfläche. Man stelle zu diesem Ende folgende, noch auf einen elastischen Körper von beliebiger Form bezügliche Betrachtung an. Man denke sich in dem Körper in seinem ursprünglichen Zustande eine unendlich klefne Kugel und in derselben einen Durchmesser $a$ und eine auf diesem senkrechte Diametral-Ebene $\boldsymbol{A}$; bei der Form-Änderung geht die Kugel in ein Ellipsoïd über; die Molecülen, die anf $a$ und auf $\boldsymbol{A}$ liegen, liegen dann auf 
einem Durchmesser $\boldsymbol{a}^{\prime}$ und auf einer Diametral-Ebene $\boldsymbol{A}^{\prime}$ des Ellipsoïds, welche conjugirt zu einander sind. Im Allgemeinen wird daher $a^{\prime}$ nicht senkrecht auf $\boldsymbol{A}^{\prime}$ sein. Ist $\boldsymbol{a}^{\prime}$ senkrecht auf $\boldsymbol{A}^{\prime}$, so wird $\boldsymbol{a}^{\prime}$ gleich einer Haupt-Axe des Ellipsoïds sein, und das Maximum und das Minimum der auf $\boldsymbol{a}^{\prime}$ senkrechten Durchmesser werden gleich den beiden andern Haupt-Axen sein; d. h. es wird die Dilatation von $a^{\prime}$ die eine Hauptdilatation, und die beiden andern Hauptdilatationen werden das Maximum und das Minimum der auf $a^{\prime}$ senkrechten Dilatationen sein.

Wendet man diesen Satz auf den Fall der Platte an, so folgt daraus bei Berücksichtigung der Annahme (1.): dafs für irgend einen Punot im Innern der Platte die Dilatation in der Richtung der durch ihn gezogenen Normale der Mittelfläche eine der Hauptdilatationen ist. Wir wollen z die ursprüngliche Entfernung des betrachteten Puncts von der Mittelfläche, $z^{\prime}$ seine Entfernung von derselben nach der Form-Änderung nennen; gleichzeitig möge durch $\boldsymbol{z}^{\prime}$ auch die Normale der Mittelfläche nach der Form-Änderung in Rücksicht auf ihre Lage bezeichnet werden. Setzt man

$$
\mathfrak{z}^{\prime}-\mathfrak{z}=q \text {, }
$$

so wird also $\frac{\partial q}{\partial z}$ der Werth einer Hauptdilatation sein. Da diese unendlich klein sein soll, und da $q$ gleichzeitig mit $z$ verschwindet, so mufs $q$ gegen $z$ unendlich klein sein.

Bei Berücksichtigung der Annahme (2.) sieht man, dafs die Dilatation in irgend einer auf $z^{\prime}$ senkrechten Richtung $=\frac{z^{\prime}}{\varrho}$ ist, wenn $\varrho$ den Krümmungsradius der Curve, in welcher die durch $z^{\prime}$ und die betreffende Richtung gelegte Ebene die Mittelfläche schneidet, für den Fufspunct von $z^{\prime}$ bezeichnet. Nennt man daher die Krümmungsradien der Hauptschnitte der Mittelfläche für den Fufspunct von $z^{\prime}$, $\varrho_{1}$ und $\varrho_{2}$, so sind $\frac{z^{\prime}}{\varrho_{1}}$ und $\frac{z^{\prime}}{\varrho_{2}}$ die Werthe der beiden andern Hauptdilatationen. Da $z^{\prime}-z$ unendlich klein gegen $z$ ist, so kann man für diese auch $\frac{z}{\varrho_{1}}$ und $\frac{z}{\varrho_{2}}$ schreiben.

Die Werthe der Hauptdilatationen $\lambda_{1}, \lambda_{2}, \lambda_{3}$, die jetzt gefunden wurden, substituire man in die Gleichung (1.). Drückt man dann noch das Element des Volumens der Platte durch $d f . d z$ aus, indem man unter $d f$ das Element der Mittelfläche versteht, so erhält man die Gleichung

$$
0=\delta \boldsymbol{P}-\boldsymbol{K} \delta \iint d f d z\left\{\left(\frac{\partial q}{\partial z}\right)^{2}+\left(\frac{z}{\varrho_{1}}\right)^{2}+\left(\frac{z}{\varrho_{2}}\right)^{2}+\theta\left(\frac{\partial q}{\partial z}+\frac{z}{\varrho_{1}}+\frac{z}{\varrho_{2}}\right)^{2}\right\} .
$$


Bezeichnet man die Dicke der Platte durch $2 \varepsilon$, so ist die Integration in Bezug auf $z$ von $z=-\varepsilon$ bis $z=\varepsilon$ zu nehmen.

Wir wollen jetzt zeigen, dafs sich die eine der Hauptdilatationen $\frac{\partial q}{\partial z}$ durch die beiden andern $\frac{z}{\varrho_{1}}$ und $\frac{z}{\varrho_{2}}$ ausdrücken läfst, ohne dafs dazu die Kenntnifs der Kräfte nöthig wäre, welche die Form-Änderung der Platte bewirkt haben. Es ist zu dem Ende der Werth von $\delta \boldsymbol{P}$, der durch die Gleichung (2.) gegeben ist, näher untersuchen. Das rechtwinklige Coordinatensystem, auf welches sich diese Gleichung bezieht, wähle man so, dafs die $x y$ Ebene die Mittelfläche in dem natürlichen Zustande der Platte ist; dann behält $\approx$ die Bedeutung, die ihm hier gegeben wurde. Man bezeichne die Winkel, welche die Normale der Mittelfläche $z^{\prime}$ mit den Coordinaten-Axen bildet, durch $\left(\boldsymbol{z}^{\prime}, \boldsymbol{x}\right),\left(\boldsymbol{z}^{\prime}, \boldsymbol{y}\right),\left(\boldsymbol{z}^{\prime}, \boldsymbol{z}\right)$, die ursprünglichen Coordinaten des Fufspuncts von $\boldsymbol{z}^{\prime}$ durch $x_{0}, y_{0}, 0$, die Verrückungen, die dieser Punct in den Richtungen der Axen erlitten hat, durch $u_{0}, v_{0}, w_{0}$, so ist der Annahme (1.) zufolge:

$$
\begin{aligned}
x+u & =x_{0}+u_{0}+z^{\prime} \cos \left(z^{\prime}, x\right), \\
y+v & =y_{0}+v_{0}+z^{\prime} \cos \left(z^{\prime}, y\right), \\
z+w & =w_{0}+z^{\prime} \cos \left(z^{\prime}, z\right),
\end{aligned}
$$

oder, da $z^{\prime}-z$ unendlich klein gegen $z$ ist:

$$
\begin{aligned}
x+u & =x_{0}+u_{0}+z \cos \left(z^{\prime}, x\right), \\
y+v & =y_{0}+v_{0}+z \cos \left(z^{\prime}, y\right), \\
z+u & =\boldsymbol{w}_{0}+z \cos \left(\boldsymbol{z}^{\prime}, \boldsymbol{z}\right) .
\end{aligned}
$$

Hieraus ergiebt sich:

$$
\text { (8.) } \quad\left\{\begin{array}{l}
\delta \boldsymbol{u}=\delta \boldsymbol{u}_{0}+\boldsymbol{z} \delta \cos \left(\boldsymbol{z}^{\prime}, \boldsymbol{x}\right), \\
\delta \boldsymbol{v}=\delta \boldsymbol{v}_{0}+\boldsymbol{z} \delta \cos \left(\boldsymbol{z}^{\prime}, y\right), \\
\delta \boldsymbol{w}=\delta \boldsymbol{w}_{0}+\boldsymbol{z} \delta \cos \left(\boldsymbol{z}^{\prime}, \boldsymbol{z}\right) .
\end{array}\right.
$$

Diese Werthe von $\delta u, \delta v$, $\delta w$ sind in die Gleichung (2.) zu subsituiren. Die zweiten Theile derselben sind, da sie den Factor $z$ enthalten, unendlich klein gegen die ersten und können daher gegen diese im Allgemeinen vernachlässigt werden; wir haben sie beibehalten, um den Fall von den obigen Betrachtungen nicht auszuschliefsen, indem die Integrale

$\int_{-\varepsilon}^{+\varepsilon} \boldsymbol{X} \boldsymbol{z} d \boldsymbol{z}, \int_{-\varepsilon}^{+\varepsilon} \boldsymbol{Y} z d z, \int_{-\varepsilon}^{+\varepsilon} \boldsymbol{Z} z d z, \int_{-\varepsilon}^{+\varepsilon}(\boldsymbol{X}) \approx d z, \int_{-\varepsilon}^{+\varepsilon}(\boldsymbol{Y}) z d z, \int_{-\varepsilon}^{+\varepsilon}(\boldsymbol{Z}) z d z$ von derselben Ordnung sind wie die Integrale

$$
\int_{-\varepsilon}^{+\varepsilon} \boldsymbol{X} d \boldsymbol{z}, \int_{-\varepsilon}^{+\varepsilon} \boldsymbol{Y} d z, \int_{-\varepsilon}^{+\varepsilon} \boldsymbol{Z} d z, \int_{-\varepsilon}^{+\varepsilon}(\boldsymbol{X}) d z, \int_{-\varepsilon}^{+\varepsilon}(\boldsymbol{Y}) d z, \int_{-\varepsilon}^{+\varepsilon}(\boldsymbol{Z}) d z
$$


Stellt man sich die Werthe von $\delta u, \delta v, \delta w$ aus (8.) in (2.) substituirt vor, so sieht man, dafs $\delta \boldsymbol{P}$ unabhängig von $\delta q$ ist; es folgt daraus, dafs das von $\delta q$ abhängige Glied des zweiten Theils der Gleichung (7.) für sich verschwinden mufs. Daraus folgt

oder

$$
(1+\theta) \frac{\partial q}{\partial z}+\theta\left(\frac{z}{\varrho_{1}}+\frac{z}{\varrho_{2}}\right)=0
$$

$$
\frac{\partial q}{\partial z}=-\frac{\theta}{1+\theta}\left(\frac{z}{\varrho_{1}}+\frac{z}{\varrho_{2}}\right)
$$

Setzt man diesen Werth von $\frac{\partial q}{\partial z}$ in die Gleichung (7.), so giebt sie:

$$
0=\delta \boldsymbol{P}-\boldsymbol{K} \delta \iint d f d z z^{2}\left\{\frac{1}{\varrho_{1}^{2}}+\frac{1}{\varrho_{2}^{2}}+\frac{\theta}{1+\theta}\left(\frac{1}{\varrho_{1}}+\frac{1}{\varrho_{2}}\right)^{2}\right\},
$$

oder, wenn man die Integration nach $z$ ausführt:

(9.) $\quad 0=\delta \boldsymbol{P}-\frac{2}{3} \varepsilon^{3} \boldsymbol{K} \delta \int d f\left(\frac{1}{\varrho_{1}^{2}}+\frac{1}{\varrho_{2}^{2}}+\frac{\theta}{1+\theta}\left(\frac{1}{\varrho_{1}}+\frac{1}{\varrho_{2}}\right)^{2}\right)$.

S. 3.

Die gefundene allgemeine Gleichgewichtsbedingung für eine Platte wollen wir nun auf den Fall anwenden, den Poisson behandelt hat: auf den Fall nämlich, dafs die Platte sich nur unendlich wenig von ihrer ursprünglichen Gleichgewichtslage entfernt hat. Wir beginnen mit der weitern Entwicklung des Werths von $\delta \boldsymbol{P}$.

Ist $w_{0}$ eine unendlich kleine Gröfse erster Ordnung, so werden, da die Mittelfläche keine Dilatationen erlitten haben soll, $\boldsymbol{u}_{0}$ und $\boldsymbol{v}_{0}$ unendlich kleine Gröfsen zweiter Ordnung sein; daher lassen sich $\delta u_{0}$ und $\delta v_{0}$ als unendlich klein gegen $\delta w_{0}$ betrachten und die Gleichungen (8.) wie folgt schreiben:

$$
\begin{array}{ll}
\delta \boldsymbol{u}= & \boldsymbol{z} \delta \cos \left(\boldsymbol{z}^{\prime}, \boldsymbol{x}\right), \\
\delta \boldsymbol{v}= & \boldsymbol{z} \delta \cos \left(\boldsymbol{z}^{\prime}, \boldsymbol{y}\right), \\
\delta \boldsymbol{w}=\delta \boldsymbol{w}_{0}+\boldsymbol{z} \delta \cos \left(\boldsymbol{z}^{\prime}, \boldsymbol{z}\right) .
\end{array}
$$

Ferner ist in dem vorliegenden Falle, wenn man wiederum nur unendlich kleine Gröfsen erster Ordnung berücksichtigt:

$$
\cos \left(z^{\prime}, x\right)=-\frac{\partial w_{0}}{\partial x_{0}}, \quad \cos \left(z^{\prime}, y\right)=-\frac{\partial w_{0}}{\partial y_{0}}, \quad \cos \left(z^{\prime}, z\right)=1
$$

und daher wird:

$$
\delta \boldsymbol{u}=-z \frac{\partial \delta w_{0}}{\partial x_{0}}, \quad \delta v=-z \frac{\partial \delta w_{0}}{\partial y_{0}}, \quad \delta \boldsymbol{w}=\delta w_{0} .
$$

Diese Werthe substituire man in die Gleichung (2.). Drückt man dann wieder 
64 4. Kirchhoff, üb. d. Gleichgewicht u.d. Bewegung einer elastischen Scheibe.

das Element des Volumens der Platte durch $d f d z$ aus und das Element der Oberfläche ihres Randes durch $d s d z$, indem man unter $d s$ das Element der Contour ihrer Mittelfläche versteht, und schreibt der Bequemlichkeit wegen $w, x, y$ statt $w_{0}, x_{0}, y_{0}$, so ergiebt sich:

$$
\begin{aligned}
\delta \boldsymbol{P}= & \iint d f d z\left\{\boldsymbol{Z} \delta w-z\left(\boldsymbol{X} \frac{\partial \delta w}{\partial x}+\boldsymbol{Y} \frac{\partial \delta w}{\partial y}\right)\right\} \\
& +\iint d s d x\left\{(\boldsymbol{Z}) \delta w-z\left((\boldsymbol{X}) \frac{\partial \delta w}{\partial x}+(\boldsymbol{Y}) \frac{\partial \delta w}{\partial z}\right)\right\} .
\end{aligned}
$$

Die Kräfte $\boldsymbol{X}, \boldsymbol{Y}, \boldsymbol{Z}$ und die Druckkräfte $(\boldsymbol{X}),(\boldsymbol{Y}),(\boldsymbol{Z})$ lassen sich hier als unabhängig von $w$ betrachten, weil $\boldsymbol{w}$ unendlich klein sein soll. Den Ausdruck von $\delta \boldsymbol{P}$ wollen wir noch umformen. Er läfst sich zunächst auf folgende Weise schreiben:

$$
\begin{aligned}
\delta \boldsymbol{P}= & \iiint d x d y d z\left\{\boldsymbol{Z}+\boldsymbol{z}\left(\frac{\partial \boldsymbol{X}}{\partial x}+\frac{\partial \boldsymbol{Y}}{\partial y}\right)\right\} \delta w \\
& -\iiint z d x d y d z \frac{\partial \boldsymbol{X} \delta w}{\partial x}-\iiint z d x d y d z \frac{\partial \boldsymbol{Y} \delta w}{\partial y} \\
& +\iiint d s d z\left\{(\boldsymbol{Z}) \delta w-z\left((\boldsymbol{X}) \frac{\partial \delta w}{\partial x}+(\boldsymbol{Y}) \frac{\partial \delta w}{\partial y}\right)\right\} .
\end{aligned}
$$

Das zweite und dritte dieser vier Integrale läfst sich umgestalten durch Anwendung der Formeln:

$$
\left\{\begin{array}{l}
\int d x d y \frac{\partial F}{\partial x}=-\int d s \cos \varphi \boldsymbol{F}, \\
\int d x d y \frac{\partial F}{\partial y}=-\int d s \sin \varphi \boldsymbol{F} .
\end{array}\right.
$$

In denselben bedeutet $\boldsymbol{F}$ eine beliebige Function von $x$ und $y$; die zweifache Integration ist über eine begrenzte Fläche, die einfache über die Contour derselben auszudehnęn; $\varphi$ bezeichnet denjenigen Winkel, den die nach dem Innern der begrenzten Fläche gerichtete Normale der Contour mit der positiven $x$ Axe bildet, und den diese Axe beschreibt, wenn sie in derjenigen Richtung gedreht wird (bis sie jener Normale parallel ist), in der sie gedreht werden mufs, damit sie nach einer Drehung um 90' die Lage der positiven $y$ Axe einnimmt. Durch Benutzung dieser Formeln erhält man für $\delta \boldsymbol{P}$ folgenden Werth:

$$
\begin{aligned}
\delta \boldsymbol{P} & =\iiint d x d y d z\left\{\boldsymbol{Z}+\boldsymbol{z}\left(\frac{\partial \boldsymbol{X}}{\partial x}+\frac{\partial \boldsymbol{Y}}{\partial y}\right)\right\} \delta w \\
& +\iint d s d z\{(\boldsymbol{Z})+\boldsymbol{z}(\boldsymbol{X} \cos \varphi+\boldsymbol{Y} \sin \varphi)\} \delta w \\
& -\iint z d s d z\left\{(\boldsymbol{X}) \frac{\partial \delta w}{\partial x}+(\boldsymbol{Y}) \frac{\partial \delta w}{\partial y}\right\} .
\end{aligned}
$$


In den letzten dieser drei Theile von $\delta \boldsymbol{P}$ führe man an die Stelle von $\frac{\partial \delta w}{\partial x}$ und $\frac{\partial \delta w}{\partial y}$ den Differentialquotienten von $\delta w$ nach der nach Innen gerichteten Normale der Contour, $\frac{\partial \delta w}{\partial N}$, und den Differentialquotienten von $\delta w$ nach dem Bogen der Contour, $\frac{\partial \delta w}{\partial s}$, ein. Man nehme den Bogen $s$ in der Richtung als so wachsend an, dafs der Winkel, den die in der Richtung des wachsenden Bogens gezogene Tangente mit der positiven $x$ Axe bildet, und den diese Axe beschreibt, wenn sie in der Weise gedreht wird, die bei der Definition von $\varphi$ bezeichnet worden ist, bis sie der Tangente parallel wird, $=\varphi-90^{\circ}$ ist. Dann ergeben sich folgende Gleichungen:

$$
\text { (11.) } \quad\left\{\begin{array}{l}
\frac{\partial \delta w}{\partial x}=\frac{\partial \delta w}{\partial N} \cos \varphi+\frac{\partial \delta w}{\partial s} \sin \varphi \\
\frac{\partial \delta w}{\partial y}=\frac{\partial \delta w}{\partial N} \sin \varphi-\frac{\partial \delta w}{\partial s} \cos \varphi
\end{array}\right.
$$

Es wird daher:

$$
\begin{aligned}
& \iint z d s d z\left\{(\boldsymbol{X}) \frac{\partial \delta w}{\partial x}+(\boldsymbol{Y}) \frac{\partial \delta w}{\partial y}\right\} \\
= & \iint z d s d z\{(\boldsymbol{X}) \cos \varphi+(\boldsymbol{Y}) \sin \varphi\} \frac{\partial \delta w}{\partial \boldsymbol{N}} \\
+ & \iint z d s d z\{(\boldsymbol{X}) \sin \varphi-(\boldsymbol{Y}) \cos \varphi\} \frac{\partial \delta w}{\partial s} .
\end{aligned}
$$

Das zweite der Integrale auf der rechten Seite dieser Gleichung stellen wir uns partiell nach $s$ integrirt vor; dabei verschwindet das aus dem Integralzeichen hervortretende Glied, indem die Integration sich auf eine geschlossene Curve bezieht; dann substituire man für die linke Seite der Gleichung die rechte in den Ausdruck von $\delta \boldsymbol{P}$. Dies giebt

$$
\begin{aligned}
\delta \boldsymbol{P} & =\iiint d x d y d z\left\{\boldsymbol{Z}+\boldsymbol{z}\left(\frac{\partial \boldsymbol{X}}{\partial x}+\frac{\partial \boldsymbol{Y}}{\partial y}\right)\right\} \delta w \\
& +\iint d s d z\left\{(\boldsymbol{Z})+z \frac{\partial((\boldsymbol{X}) \sin \varphi-(\boldsymbol{Y}) \cos \varphi)}{\partial s}+z(\boldsymbol{X} \cos \varphi+\boldsymbol{Y} \sin \varphi)\right\} \delta \boldsymbol{W} \\
& -\iint d s d z z\{(\boldsymbol{X}) \cos \varphi+(\boldsymbol{Y}) \sin \varphi\} \frac{\partial \delta w}{\partial \boldsymbol{N}}
\end{aligned}
$$

Nun bilde man den zweiten Theil der rechten Seite der Gleichung (9.). Wir stellen uns durch einen Punct der Mittelfläche, der die Coordinaten $x, y, w$ hat, eine Ebene gelegt vor, die der $\approx$ Axe parallel ist und mit der $x \approx$ Ebene den Winkel $\vartheta$ bildet; $\rho$ sei der Krümmungsradius des Schnittes dieser Ebene 
66 4. Kirchhoff, üb. d. Gleichgewicht u. d Bewegung einer elastischen Scheibe.

und der Mittelfläche für den Punct $(x, y, w)$, so ist

$$
\frac{1}{\varrho}=\frac{\partial^{2} w}{\partial x^{2}} \cos ^{2} \vartheta+2 \frac{\partial^{2} w}{\partial x \partial y} \cos \vartheta \sin \vartheta+\frac{\partial^{2} w}{\partial y^{2}} \sin ^{2} \vartheta
$$

Die Werthe von $\frac{1}{\varrho_{1}}$ und $\frac{1}{\varrho_{2}}$ sind das Maximum und das Minimum von $\frac{1}{\varrho}$; sie sind daher die Wurzeln der Gleichung

$$
\left(\frac{\partial^{2} w}{\partial x^{2}}-\Lambda\right)\left(\frac{\partial^{2} w}{\partial y^{2}}-\Lambda\right)-\left(\frac{\partial^{2} w}{\partial x \partial y}\right)^{2}=0 .
$$

Hieraus folgt:

$$
\begin{aligned}
& \frac{1}{\varrho_{1}}+\frac{1}{\varrho_{2}}=\frac{\partial^{2} w}{\partial x^{2}}+\frac{\partial^{2} w}{\partial y^{2}}, \\
& \frac{1}{\varrho_{1}^{2}}+\frac{1}{\varrho_{2}^{2}}=\left(\frac{\partial^{2} w}{\partial x^{2}}\right)^{2}+2\left(\frac{\partial^{2} w}{\partial x \partial y}\right)^{2}+\left(\frac{\partial^{2} w}{\partial y^{2}}\right)^{2} .
\end{aligned}
$$

Diese Werthe sind in die Gleichung (9.) zu substituiren. Man setze

$$
\left\{\begin{array}{l}
\boldsymbol{Q}=\iint \boldsymbol{d} x d y\left(\left(\frac{\partial^{2} w}{\partial x^{2}}\right)^{2}+2\left(\frac{\partial^{2} w}{\partial x \partial y}\right)^{2}+\left(\frac{\partial^{2} w}{\partial y^{2}}\right)^{2}\right), \\
\boldsymbol{R}=\iint d x d y\left(\frac{\partial^{2} w}{\partial x^{2}}+\frac{\partial^{2} w}{\partial y^{2}}\right)^{2}
\end{array}\right.
$$

so giebt die Gleichung (9.)

$$
\text { (14.) } \delta \boldsymbol{P}-\frac{2}{3} \varepsilon^{3} \boldsymbol{K}\left(\delta \boldsymbol{Q}+\frac{\theta}{1+\theta} \delta \boldsymbol{R}\right)=0 \text {. }
$$

Nun ist Bildung von $\delta \boldsymbol{Q}$ und $\delta \boldsymbol{R}$ nöthig. Es findet sich

$$
\text { (15.) } \quad \delta \boldsymbol{Q}=2 \iint d \boldsymbol{x} d y\left\{\frac{\partial^{2} w}{\partial x^{2}} \frac{\partial^{2} \delta w}{\partial \boldsymbol{x}^{2}}+2 \frac{\partial^{2} w}{\partial x \partial y} \frac{\partial^{2} \delta w}{\partial x \partial y}+\frac{\partial^{2} w}{\partial \boldsymbol{y}^{2}} \frac{\partial^{2} \delta w}{\partial y^{2}}\right\}:
$$

Es ist aber:

$$
\begin{gathered}
\iint d x d y \frac{\partial^{2} w}{\partial x^{2}} \frac{\partial^{2} \delta w}{\partial x^{2}} \\
=\iint d x d y \frac{\partial \frac{\partial^{2} w}{\partial x^{2}} \frac{\partial \delta w}{\partial x}}{\partial x}-\iint d x d y \frac{\partial \frac{\partial^{3} w}{\partial x^{3}} \delta w}{\partial x}+\iint d x d y \frac{\partial^{4} w}{\partial x^{4}} \delta w \\
\iint d x d y \frac{\partial^{2} w}{\partial x \partial y} \frac{\partial^{2} \delta w}{\partial x \partial y} \\
=\iint d x d y \frac{\partial \frac{\partial^{2} w}{\partial x \partial y} \frac{\partial \delta w}{\partial y}}{\partial x}-\iint d x d y \frac{\partial \frac{\partial^{3} w}{\partial x^{2} \partial y} \delta w}{\partial y}+\iint d x d y \frac{\partial^{4} w}{\partial x^{2} \partial y^{2}} \delta w
\end{gathered}
$$

und auch 


$$
\begin{aligned}
& \int d x d y \frac{\partial^{2} w}{\partial x \partial y} \frac{\partial^{2} \delta w}{\partial x \partial y} \\
& =\iint d x d y \frac{\partial \frac{\partial^{2} w}{\partial x \partial y} \frac{\partial \delta w}{\partial x}}{\partial y}-\iint d x d y \frac{\partial \frac{\partial^{3} w}{\partial x \partial y^{2}} \delta w}{\partial x}+\iint d x d y \frac{\partial^{4} w}{\partial x^{2} \partial y^{2}} \delta w, \\
& \iint d x d y \frac{\partial^{2} w}{\partial y^{2}} \frac{\partial^{2} \delta w}{\partial y^{2}} \\
& =\iint d x d y \frac{\partial \frac{\partial^{2} w}{\partial y^{2}} \frac{\partial \delta w}{\partial y}}{\partial y}-\iint d x d y \frac{\partial \frac{\partial^{3} w}{\partial y^{3}} \delta w}{\partial y}+\iint d x d y \frac{\partial^{4} w}{\partial y^{4}} \delta w .
\end{aligned}
$$

Diese vier Gleichungen addire man. Auf der linken Seite der resultirenden Gleichung erhält man dann $\frac{1}{2} \delta Q$; auf der rechten Seite derselben verwandele man diejenigen Integrale, welche unter den Integralzeichen das Element der Fläche mit einem nach $\boldsymbol{x}$ oder nach $\boldsymbol{y}$ genommenen Differentialquotienten multiplicirt enthalten, mit Hülfe der Formeln (10.) in Integrale, die sich auf die Contour der Mittelfläche beziehen. In einem Theile dieser Integrale kommen die Differentialquotienten $\frac{\partial \delta w}{\partial x}$ und $\frac{\partial \delta w}{\partial y}$ vor; diese drücke man mit Hülfe der Gleichungen (11.) durch $\frac{\partial \delta w}{\partial \boldsymbol{N}}$ und $\frac{\partial \delta w}{\partial s}$ aus und integrire die Glieder, welche $\frac{\partial \delta w}{\partial s}$ enthalten, partiell nach $s$. Die Glieder, welche hierbei vor die Integralzeichen treten, verschwinden, weil die Integration sich auf eine geschlossene Curve bezieht und es ergiebt sich:

$$
\begin{aligned}
\delta \boldsymbol{Q}= & 2 \iint d x d y\left\{\frac{\partial^{4} w}{\partial x^{4}}+2 \frac{\partial^{4} w}{\partial x^{2} \partial y^{2}}+\frac{\partial^{4} w}{\partial y^{4}}\right\} \delta \boldsymbol{w} \\
& +2 \int d s\left\{\left(\frac{\partial^{3} w}{\partial x^{3}}+\frac{\partial^{3} w}{\partial x}\right) \cos \varphi+\left(\frac{\partial^{3} w}{\partial x^{2} \partial y}+\frac{\partial^{3} w}{\partial y^{3}}\right) \sin \varphi\right. \\
& \left.-\frac{\partial\left(\frac{\partial^{2} w}{\partial x \partial y}\left(\cos ^{2} \varphi-\sin ^{2} \varphi\right)+\left(\frac{\partial^{2} w}{\partial y^{2}}-\frac{\partial^{2} w}{\partial x^{2}}\right) \cos \varphi \sin \varphi\right)}{\partial s}\right\} \delta w \\
& -2 \int d s\left\{\frac{\partial^{2} w}{\partial x^{2}} \cos ^{2} \varphi+2 \frac{\partial^{2} w}{\partial x \partial y} \cos \varphi \sin \varphi+\frac{\partial^{2} w}{\partial y^{2}} \sin ^{2} \varphi\right\} \frac{\partial \delta w}{\partial N} .
\end{aligned}
$$

Ferner ist

$$
\text { (17.) } \quad \delta \boldsymbol{R}=2 \iint d x d y\left(\frac{\partial^{2} w}{\partial x^{2}}+\frac{\partial^{2} w}{\partial y^{2}}\right)\left(\frac{\partial^{2} \delta w}{\partial x^{2}}+\frac{\partial^{2} \delta w}{\partial y^{2}}\right) \text {. }
$$

Es ist aber, wenn $\boldsymbol{F}$ und $\boldsymbol{G}$ zwei beliebige Functionen von $\boldsymbol{x}$ und $\boldsymbol{y}$ bezeichnen:

$$
\begin{gathered}
\iint d x d y \boldsymbol{F}\left(\frac{\partial^{2} \boldsymbol{G}}{\partial x^{2}}+\frac{\partial^{2} \boldsymbol{G}}{\partial y^{2}}\right) \\
=\iint d x d y \boldsymbol{G}\left(\frac{\partial^{2} \boldsymbol{F}}{\partial x^{2}}+\frac{\partial^{2} \boldsymbol{F}}{\partial y^{2}}\right)+\int d s \frac{\partial \boldsymbol{F}}{\partial \boldsymbol{N}} \boldsymbol{G}-\int d s \boldsymbol{F} \frac{\partial \boldsymbol{G}}{\partial \boldsymbol{N}}
\end{gathered}
$$


setzt man also

$$
\boldsymbol{F}=\frac{\partial^{2} w}{\partial x^{2}}+\frac{\partial^{2} w}{\partial y^{2}}, \quad \boldsymbol{G}=\delta w
$$

und berücksichtigt, dafs

$$
\frac{\partial \boldsymbol{F}}{\partial \boldsymbol{N}}=\frac{\partial \boldsymbol{F}}{\partial x} \cos \varphi+\frac{\partial \boldsymbol{F}}{\partial y} \sin \varphi
$$

ist, was aus den Gleichungen (11.) folgt; so ergiebt sich

$$
\begin{aligned}
\delta \boldsymbol{R}=2 \iint d x d y & \left\{\frac{\partial^{4} w}{\partial x^{4}}+2 \frac{\partial^{4} w}{\partial x^{2} \partial y^{2}}+\frac{\partial^{4} w}{\partial y^{4}}\right\} \delta w \\
& +2 \int d s\left\{\left(\frac{\partial^{3} w}{\partial x^{3}}+\frac{\partial^{3} w}{\partial x \partial y^{2}}\right) \cos \varphi+\left(\frac{\partial^{3} w}{\partial x^{2} \partial y}+\frac{\partial^{3} w}{\partial y^{3}}\right) \sin \varphi\right\} \delta w \\
& -2 \int d s\left\{\frac{\partial^{2} w}{\partial x^{2}}+\frac{\partial^{2} w}{\partial y^{2}}\right\} \frac{\partial \delta w}{\partial \boldsymbol{N}} .
\end{aligned}
$$

Mit Hülfe der Gleichungen (12., 16. und 18.) bilde man nun die Gleichung (14.); die linke Seite derselben läfst sich darstellen als die Summe von drei Integralen, von denen das erste über die Mittelfläche selbst, die beiden andern über die Contour derselben auszudehnen sind, und von denen die beiden ersten unter den Integralzeichen den Factor $\delta w$ haben, das letzte den Factor $\frac{\partial \delta w}{\partial \boldsymbol{N}}$ hat. Den Principien der Variationsrechnung gemäfs müssen die Gröfsen, mit denen $\delta w$ und $\frac{\partial \delta w}{\partial N}$ multiplicirt vorkommen, verschwinden. Man erhält demnach die partielle Differentialgleichung

$$
\text { (19.) } \begin{aligned}
& \int_{-\varepsilon}^{+\varepsilon} \boldsymbol{Z} d z+\frac{\partial}{\partial x} \int_{-\varepsilon}^{+\varepsilon} \boldsymbol{X} z d z+\frac{\partial}{\partial y} \int_{-\varepsilon}^{+\varepsilon} Y_{z d z} \\
= & \frac{4}{3} \varepsilon^{3} \boldsymbol{K} \frac{1+2 \theta}{1+\theta}\left(\frac{\partial^{4} w}{\partial x^{4}}+2 \frac{\partial^{4} w}{\partial x^{2} \partial y^{2}}+\frac{\partial^{4} w}{\partial y^{4}}\right)
\end{aligned}
$$

und die beiden Grenzbedingungen

$$
\text { (20.) } \begin{gathered}
\int_{\varepsilon}^{+\varepsilon}(\boldsymbol{Z}) d z+\frac{\partial}{\partial s}\left(\sin \varphi \int_{\varepsilon}^{+\varepsilon}(\boldsymbol{X}) z d z-\cos \varphi \int_{-\varepsilon}^{+\varepsilon}(\boldsymbol{Y}) \boldsymbol{z} d z\right) \\
+\cos \varphi \int_{-\varepsilon}^{+\varepsilon} \boldsymbol{X} \boldsymbol{z} d z+\sin \varphi \int_{-\varepsilon}^{+\varepsilon} \boldsymbol{Y} z d z \\
=\frac{4}{3} \varepsilon^{3} \boldsymbol{K}\left\{\frac{1+2 \theta}{1+\theta}\left(\left(\frac{\partial^{3} w}{\partial x^{3}}+\frac{\partial^{3} w}{\partial x \partial y^{2}}\right) \cos \varphi+\left(\frac{\partial^{3} w}{\partial x^{2} \partial y}+\frac{\partial^{3} w}{\partial y^{3}}\right) \sin \varphi\right)\right. \\
\left.-\frac{\partial}{\partial s}\left(\frac{\partial^{2} w}{\partial x \partial y}\left(\cos ^{2} \varphi-\sin ^{2} \varphi\right)+\left(\frac{\partial^{2} w}{\partial y^{2}}-\frac{\partial^{2} w}{\partial x^{2}}\right) \cos \varphi \sin \varphi\right)\right\},
\end{gathered}
$$


4. Kirchhoff, ïb. d. Gleichgewicht u.d. Bewegung einer elastischen Scheibe.

$$
\begin{gathered}
\text { (21.) } \cos \varphi \int_{-\varepsilon}^{+\varepsilon}(\boldsymbol{X}) \approx d z+\sin \varphi \int_{-\varepsilon}^{+\varepsilon}(\boldsymbol{Y}) z d z \\
=\frac{4}{3} \varepsilon^{3} \boldsymbol{K}\left\{\frac{\theta}{1+\theta}\left(\frac{\partial^{2} w}{\partial x^{2}}+\frac{\partial^{2} w}{\partial y^{2}}\right)+\frac{\partial^{2} w}{\partial x^{2}} \cos ^{2} \varphi+2 \frac{\partial^{2} w}{\partial x \partial y} \cos \varphi \sin \varphi+\frac{\partial^{2} w}{\partial y^{2}} \sin ^{2} \varphi\right\} .
\end{gathered}
$$

Die Gleichung (19.) stimmt mit der partiellen Differentialgleichung, welche Poisson abgeleitet hat, überein; abgesehen davon, dafs Poisson $\theta=\frac{1}{2}$ gesetzt hat, während hier $\theta$ unbestimmt gelassen ist. Die drei Grenzbedingungen von Poisson lassen sich darstellen durch die Gleichungen $(20 ., 21$.$) und die Gleichung$

$$
\begin{gathered}
\text { (22.) } \sin \varphi \int_{-\varepsilon}^{+\varepsilon}(\boldsymbol{X}) \approx d z-\cos \varphi \int_{-\varepsilon}^{+\varepsilon}(\boldsymbol{Y}) z d z \\
=-\frac{4}{3} \varepsilon^{3} \boldsymbol{K}\left(\frac{\partial^{2} w}{\partial x \partial y}\left(\cos ^{2} \varphi-\sin ^{2} \varphi\right)+\left(\frac{\partial^{2} w}{\partial y^{2}}-\frac{\partial^{2} w}{\partial x^{2}}\right) \cos \varphi \sin \varphi\right) .
\end{gathered}
$$

Ich werde jetzt nachweisen, dafs $w$ bis auf eine additive lineare Function von $x$ und $y$, die willkürlich bleibt, durch die Gleichungen $(19 ., 20 ., 21$. bestimmt ist: Daraus folgt dann, dafs den Poissonschen Gleichungen nur in den speciellen Fällen genügt werden kann, in welchen die gegebenen Kräfte von der Art sind, dafs die Gleichung (22.) von selbst erfüllt wird, sobald die Gleichungen (19., 20., 21.) erfüllt sind.

Es seien $w_{1}$ und $w_{2}$ zwei Functionen, die, statt $w$ gesetzt, den Gleichungen (19., 20., 21.) genügen; dann erfüllt $w_{1}-w_{2}=w$ die Gleichungen, die aus den eben genannten entstehen, wenn an die Stelle der linken Theile derselben 0 gesetzt wird. Ich werde beweisen, dafs diesen Gleichungen nur durch eine lineare Function von $x$ und $y$ genügt werden kann.

Man stelle sich den Werth von

$$
\delta \boldsymbol{Q}+\frac{\theta}{1+\theta} \delta \boldsymbol{R}
$$

gebildet vor: einmal mit Hülfe der Gleichungen (15. und 17.), dann mit Hülfe der Gleichungen (16. und 18.), und die beiden Ausdrücke, die man dadurch erhält, einander gleich gesetzt. In der identischen Gleichung, die man dann hat, mache man $\delta w=i w$, wobei unter $i$ eine unendlich kleine Constante verstanden wird. Läfst man darauf den Factor 2i, der sich in derselben findet, weg, so wird sie: 
70 4. Kirchhoff, üb. d. Gleichgewicht u. d. Bewegung einer elastischen Scheibe.

$$
\begin{aligned}
\iint d x d y & \left\{\left(\frac{\partial^{2} w}{\partial x^{2}}\right)^{2}+2\left(\frac{\partial^{2} w}{\partial x \partial y}\right)^{2}+\left(\frac{\partial^{2} w}{\partial y^{2}}\right)^{2}+\frac{\theta}{1+\theta}\left(\frac{\partial^{2} w}{\partial x^{2}}+\frac{\partial^{2} w}{\partial y^{2}}\right)^{2}\right\} \\
= & \iint d x d y \frac{1+2 \theta}{1+\theta}\left\{\frac{\partial^{4} w}{\partial x^{4}}+2 \frac{\partial^{4} w}{\partial x^{2} \partial y^{2}}+\frac{\partial^{4} w}{\partial y^{4}}\right\} w \\
+ & \int d s\left\{\frac{1+2 \theta}{1+\theta}\left(\left(\frac{\partial^{3} w}{\partial x^{3}}+\frac{\partial^{3} w}{\partial x \partial y^{2}}\right) \cos \varphi+\left(\frac{\partial^{3} w}{\partial x^{2} \partial y}+\frac{\partial^{3} w}{\partial y^{3}}\right) \sin \varphi\right)\right. \\
& \left.-\frac{\partial}{\partial s}\left(\frac{\partial^{2} w}{\partial x \partial y}\left(\cos ^{2} \varphi-\sin ^{2} \varphi\right)+\left(\frac{\partial^{2} w}{\partial y^{2}}-\frac{\partial^{2} w}{\partial x^{2}}\right) \cos \varphi \sin \varphi\right)\right\} w \\
& -\int d s\left\{\frac{\theta}{1+\theta}\left(\frac{\partial^{2} w}{\partial x^{2}}+\frac{\partial^{2} w}{\partial y^{2}}\right)\right. \\
& \left.\quad+\frac{\partial^{2} w}{\partial x^{2}} \cos ^{2} \varphi+2 \frac{\partial^{2} w}{\partial x \partial y} \cos \varphi \sin \varphi+\frac{\partial^{2} w}{\partial y^{2}} \sin ^{2} \varphi\right\} \frac{\partial w}{\partial N} .
\end{aligned}
$$

Genügt nun $w$ den Gleichungen, in welche die Gleichungen $(19 ., 20 ., 21$. übergehen, wenn man an Stelle ihrer linken Theile 0 setzt, so verschwindet die rechte Seite dieser Gleichung; es verschwindet also auch die linke. Diese besteht aber, da $\theta$ positiv ist, aus einer Summe von lauter positiven Gröfsen: es müssen daher alle diese Gröfsen für sich verschwinden, und also für alle Puncte der Mittelfläche der Scheibe die Gleichungen

$$
\frac{\partial^{2} w}{\partial x^{2}}=0, \quad \frac{\partial^{2} w}{\partial x \partial y}=0, \quad \frac{\partial^{2} w}{\partial y^{2}}=0
$$

erfüllt werden. Diesen Gleichungen aber kann nur durch eine lineare Function von $x$ und $y$ genügt werden.

S. 4.

Die Gleichungen (19., 20., 21.) im vorigen Paragraphen will ich nun anwenden, um die Gesetze der Schwingungen einer freien, kreisförmigen Scheibe herzuleiten. Aus denselben ergiebt sich für die Schwingungen einer beliebig gestalteten Scheibe die partielle Differentialgleichung

(1.) $\quad 0=\varrho \frac{\partial^{2} w}{\partial t^{2}}+\frac{2}{3} \frac{1+2 \theta}{1+\theta} \varepsilon^{2} \boldsymbol{K}\left(\frac{\partial^{4} w}{\partial x^{4}}+2 \frac{\partial^{4} w}{\partial x^{2} \partial y^{2}}+\frac{\partial^{4} w}{\partial y^{4}}\right)$, in welcher $\varrho$ die Dichtigkeit der Scheibe bezeichnet, nebst den Grenzbedingungen

$$
\left\{\begin{array}{c}
0=\frac{1+2 \theta}{1+\theta}\left(\left(\frac{\partial^{3} w}{\partial x^{3}}+\frac{\partial^{3} w}{\partial x \partial y^{2}}\right) \cos \varphi+\left(\frac{\partial^{3} w}{\partial x^{2} \partial y}+\frac{\partial^{3} w}{\partial y^{3}}\right) \sin \varphi\right) \\
-\frac{\partial}{\partial s}\left(\frac{\partial^{2} w}{\partial x \partial y}\left(\cos ^{2} \varphi-\sin ^{2} \varphi\right)+\left(\frac{\partial^{2} w}{\partial y^{2}}-\frac{\partial^{2} w}{\partial x^{2}}\right) \cos \varphi \sin \varphi\right), \\
0=\frac{\theta}{1+\theta}\left(\left(\frac{\partial^{2} w}{\partial x^{2}}+\frac{\partial^{2} w}{\partial y^{2}}\right)+\frac{\partial^{2} w}{\partial x^{2}} \cos ^{2} \varphi+2 \frac{\partial^{2} w}{\partial x \partial y} \cos \varphi \sin \varphi+\frac{\partial^{2} w}{\partial y^{2}} \sin ^{2} \varphi\right) .
\end{array}\right.
$$

Man erhält diese Gleichungen, indem man in jenen $(\boldsymbol{X})=(\boldsymbol{Y})=(\boldsymbol{Z})=\mathbf{0}$, 
4. Kirchh off, ̈̈̈.d. Gleichgewicht u. d. Beweguny einer elastischen Scheibe. 71 $\boldsymbol{X}=-\varrho \frac{\partial^{2} u}{\partial t^{2}}, \quad \boldsymbol{Y}=-\varrho \frac{\partial^{2} v}{\partial t^{2}}, \quad \boldsymbol{Z}=-\varrho \frac{\partial^{2} w}{\partial t^{2}}$ setzt und berücksichtigt, dafs den beiden in ( $\$$. 2.) gemachten Annahmen zufolge $\frac{\partial^{2} u}{\partial t^{2}}$ und $\frac{\partial^{2} v}{\partial t^{2}}$ nicht unendlich grofs gegen $\frac{\partial^{2} w}{\partial t^{2}}$ sein können. Zu den Bedingungen (1. und 2.) sind noch die hinzuzufügen, dafs für $t=0, w$ und $\frac{\partial w}{\partial t}$ in zwei gegebene Functionen von $x$ und $y$ übergehen; dann wird $w$ vollkommen bestimmt sein.

Wir suchen zuerst eine particuläre Lösung der Differentialgleichung (1), die die Gleichungen (2.) erfüllt. Diese wird sich dann so verallgemeinern lassen, dafs auch den Bedingungen, die für $t=0$ gelten, genügt wird.

Man setze der Kürze wegen

und

$$
\frac{2}{3} \frac{1+2 \theta}{1+\theta} \varepsilon^{2} \frac{K}{\varrho}=a^{2}
$$

$$
\text { (3.) } \quad w=u \sin \left(4 \lambda^{2} a t\right) \text {; }
$$

wo $u$ eine Function von $x$ und $y, \lambda$ eine Constante bezeichnet, über die zu verfügen wir uns vorbehalten Durch (3.) wird der Gleichung (1.) genügt werden, wenn $u$ folgende Gleichung erfüllt:

$$
\text { (4.) } 16 \lambda^{4} u=\frac{\partial^{4} u}{\partial x^{4}}+2 \frac{\partial^{4} u}{\partial x^{2} \partial y^{2}}+\frac{\partial^{4} u}{\partial y^{4}} \text {. }
$$

Diese kann ersetzt werden durch die zwei Gleichungen

Macht man

$$
\left\{\begin{array}{l}
4 \lambda^{2} v=\frac{\partial^{2} u}{\partial x^{2}}+\frac{\partial^{2} u}{\partial y^{2}} \\
4 \lambda^{2} u=\frac{\partial^{2} v}{\partial^{2} x^{2}}+\frac{\partial^{2} v}{\partial y^{2}}
\end{array}\right.
$$

(6.) $\quad \boldsymbol{u}=\boldsymbol{S}+\boldsymbol{D}, \quad \boldsymbol{v}=\boldsymbol{S}-\boldsymbol{D}$,

so folgen für $\boldsymbol{S}$ und $\boldsymbol{D}$ die Differentialgleichungen

$$
\begin{aligned}
4 \lambda^{2} \boldsymbol{S} & =\frac{\partial^{2} \boldsymbol{S}}{\partial x^{2}}+\frac{\partial^{2} \boldsymbol{S}}{\partial y^{2}}, \\
-4 \lambda^{2} \boldsymbol{D} & =\frac{\partial^{2} \boldsymbol{D}}{\partial x^{2}}+\frac{\partial^{2} \boldsymbol{D}}{\partial y^{2}} .
\end{aligned}
$$

Nun führe man an die Stelle der rechtwinkligen Coordinaten Polarcoordinaten $\boldsymbol{r}, \boldsymbol{\psi}$ ein; dann werden die letzten Gleichungen:

$$
\begin{aligned}
4 \lambda^{2} \boldsymbol{S} & =\frac{\partial^{2} \boldsymbol{S}}{\partial r^{2}}+\frac{1}{r} \frac{\partial \boldsymbol{S}}{\partial r}+\frac{1}{r^{2}} \frac{\partial^{2} \boldsymbol{S}}{\partial \psi^{2}}, \\
-4 \lambda^{2} \boldsymbol{D} & =\frac{\partial^{2} \boldsymbol{D}}{\partial r^{2}}+\frac{1}{r} \frac{\partial \boldsymbol{D}}{\partial r}+\frac{1}{r^{2}} \frac{\partial^{2} \boldsymbol{D}}{\partial \psi^{2}} .
\end{aligned}
$$


Diesen wird genügt, wenn man

$$
\text { (7.) }\left\{\begin{array}{l}
\boldsymbol{S}=\boldsymbol{A} \cdot \cos n \psi \cdot \boldsymbol{X} \\
\boldsymbol{D}=\boldsymbol{B} \cdot \cos n \psi \cdot \boldsymbol{Y}
\end{array}\right.
$$

setzt, wo $\boldsymbol{A}$ und $\boldsymbol{B}$ willkürliche Constanten, $\boldsymbol{n}$ eine ganze Zahl und $\boldsymbol{X}$ und $\boldsymbol{Y}$ zwei Functionen von $\boldsymbol{r}$ bezeichnen, die die Gleichungen

$$
\left\{\begin{array}{l}
\frac{d^{2} X}{d r^{2}}+\frac{1}{r} \frac{d X}{d r}-\left(\frac{n^{2}}{r^{2}}+4 \lambda^{2}\right) X=0 \\
\frac{d^{2} Y}{d r^{2}}+\frac{1}{r} \frac{d Y}{d r}-\left(\frac{n^{2}}{r^{2}}-4 \lambda^{2}\right) Y=0
\end{array}\right.
$$

erfüllen. Führt man

$$
\boldsymbol{x}=\lambda \boldsymbol{r}
$$

ein, so werden diese Gleichungen

$$
\left\{\begin{array}{l}
\frac{d^{2} X}{d x^{2}}+\frac{1}{x} \frac{d X}{d x}-\left(\frac{n^{2}}{x^{2}}+4\right) X=0 \\
\frac{d^{2} Y}{d x^{2}}+\frac{1}{x} \frac{d Y}{d x}-\left(\frac{n^{2}}{x^{2}}-4\right) Y=0
\end{array}\right.
$$

Particuläre Integrale sind die folgenden:

$$
\left\{\begin{array}{l}
X^{(n)}=\frac{x^{n}}{1.2 .3 \ldots n}\left(1+\frac{x^{2}}{1 . n+1}+\frac{x^{4}}{1.2 . n+1 . n+2}+\frac{x^{6}}{1.2 .3 \cdot n+1 . n+2 . n+3}+\text { etc. }\right) \\
Y^{(n)}=\frac{x^{n}}{1.2 .3 \ldots n}\left(1-\frac{x^{2}}{1 . n+1}+\frac{x^{4}}{1.2 . n+1 . n+2}-\frac{x^{6}}{1.2 .3 . n+1 . n+2 . n+3}+\text { etc. }\right)
\end{array}\right.
$$

und andere particuläre Integrale:

$$
\left\{\begin{array}{l}
\boldsymbol{X}^{(n)^{\prime}}=\boldsymbol{X}^{(n)} \int_{x_{0}}^{x} \frac{d x}{x \boldsymbol{X}^{(n)} \boldsymbol{X}^{(n)}} \\
\boldsymbol{Y}^{(n)^{\prime}}=\boldsymbol{Y}^{(n)} \int_{x_{0}}^{x} \frac{d x}{x \boldsymbol{Y}^{(n)} Y^{(n)}}
\end{array}\right.
$$

wo $x_{0}$ eine beliebige, endliche Gröfse bezeichnet. Die allgemeinen Integrale der Gleichungen (9.) sind also

$$
\begin{aligned}
& \boldsymbol{X}=\alpha \boldsymbol{X}^{(n)}+\alpha^{\prime} \boldsymbol{X}^{(n)^{\prime}}, \\
& \boldsymbol{Y}=\boldsymbol{\beta} \boldsymbol{Y}^{(n)}+\beta \boldsymbol{Y}^{(n)^{\prime}} .
\end{aligned}
$$

Aus den Gleichungen (11.) geht hervor, dafs $X^{(n)^{\prime}}$ und $Y^{(n)^{\prime}}$ für $x=0$ unendlich werden; wir nehmen an, dafs die Scheibe eine volle, keine ringförmige ist; dann müssen für $r=0, \mathrm{~d}$. h. für $x=0, u$ und $v$, also auch $\boldsymbol{X}$ und $\boldsymbol{Y}$ endlich bleiben, und daher mũssen $\alpha^{\prime}$ und $\beta^{\prime}$ verschwinden. Die Constanten $\alpha$ und $\beta$ lassen sich, ohne der Allgemeinheit zu schaden, $=1$ setzen, da wir in die Gleichungen (7.) die Constanten $\boldsymbol{A}$ und $\boldsymbol{B}$ eingeführt 
haben, und daher statt der Gleichungen (7.) schreiben:

$$
\text { (12.) } \quad\left\{\begin{array}{l}
\boldsymbol{S}=\boldsymbol{A} \cos n \psi \cdot \boldsymbol{X}^{(n)} \\
\boldsymbol{D}=\boldsymbol{B} \cos n \psi \cdot \boldsymbol{Y}^{(n)}
\end{array}\right.
$$

wo $\boldsymbol{X}^{(n)}$ und $\boldsymbol{Y}^{(n)}$ die durch die Gleichungen (10.) bestimmten Functionen bedeuten. Ich bemerke, dafs $\boldsymbol{Y}^{(n)}$ die Function ist, für welche Bessel die Bezeichnung

eingeführt hat.

$$
\boldsymbol{I}_{2 \boldsymbol{x}}^{n}
$$

Wir werden jetzt über die Constanten $A, B, \lambda$ so zu verfügen suchen, dafs den Gleichungen (2.) genügt wird. In diesen Gleichungen ist der Bogen $s$ in derjenigen Richtung als wachsend anzusehen, die bei den Gleichungen (11.) (\$. 3.) bezeichnet ist. Aus der dort gemachten Bestimmung geht hervor, dafs, wenn man $\psi$ in derjenigen Richtung wachsen läfst und den Anfangspunct von $s$ so wählt, dafs

$$
s=l \psi
$$

wird, wo $l$ den Radius der Scheibe bezeichnet,

$$
\varphi=\psi+180^{\circ}
$$

ist. Benutzt man Dies, so nehmen die Gleichungen (2.) durch Einführung der Polarcoordinaten $\boldsymbol{r}, \psi$, statt der rechtwinkligen, folgende Gestalt an:

$$
\left\{\begin{array}{l}
\frac{1+2 \theta}{1+\theta} \frac{\partial}{\partial r}\left(\frac{\partial^{2} w}{\partial r^{2}}+\frac{1}{r} \frac{\partial w}{\partial r}+\frac{1}{r^{2}} \frac{\partial^{2} w}{\partial \psi^{2}}\right)+\frac{1}{r^{2}} \frac{\partial}{\partial \psi}\left(\frac{\partial^{2} w}{\partial r \partial \psi}-\frac{1}{r} \frac{\partial w}{\partial \psi}\right)=0, \\
\frac{\theta}{1+\theta}\left(\frac{\partial^{2} w}{\partial r^{2}}+\frac{1}{r} \frac{\partial w}{\partial r}+\frac{1}{r^{2}} \frac{\partial^{2} w}{\partial \psi^{2}}\right)+\frac{\partial^{2} w}{\partial r^{2}}=0 .
\end{array}\right.
$$

Diese Gleichungen müssen für $r=l$, für alle Werthe von $\psi$ erfüllt werden, und für alle Werthe von $t$. Nach (3.) müssen daher auch die Gleichungen bestehen, welche man aus (13.) findet, wenn man $u$ statt $w$ schreibt. Diese Gleichungen geben, wenn man berücksichtigt, dafs

$$
\frac{\partial^{2} u}{\partial r^{2}}+\frac{1}{r} \frac{\partial u}{\partial r}+\frac{1}{r^{2}} \frac{\partial^{2} u}{\partial \psi^{2}}=4 \lambda^{2} v
$$

ist, was die erste der Gleichungen (5.) aussagt:

$$
\begin{aligned}
& 4 \lambda^{2} \frac{1+2 \theta}{1+\theta} \frac{\partial v}{\partial r}+\frac{1}{r^{2}} \frac{\partial^{3} u}{\partial r \partial \psi^{2}}-\frac{1}{r^{3}} \frac{\partial^{2} u}{\partial \psi^{2}}=0 \\
& 4 \lambda^{2} \frac{\theta}{1+\theta} v+\frac{\partial^{2} u}{\partial r^{2}}=0 .
\end{aligned}
$$

Führt man hier für $u$ und $v$ ihre Werthe ein, die sich aus (6. und 12.) ergeben, ersetzt $\boldsymbol{r}$ durch $\frac{x}{\lambda}$, drückt die zweiten Differentialquotienten von Crelle's Journal f. d. M. Bd. XL. Heft 1 . 
74 4. Kirchhoff, üb. d. Gleichgewicht u. d. Bewegung einer elastischen Scheibe.

$\boldsymbol{X}^{(n)}$ und $\boldsymbol{Y}^{(n)}$ mit Hülfe von (9.) durch diese Functionen selbst und ihre ersten Differentialquotienten aus und macht endlich

so ergiebt sich

$$
\frac{1+2 \theta}{1+\theta}=\gamma
$$

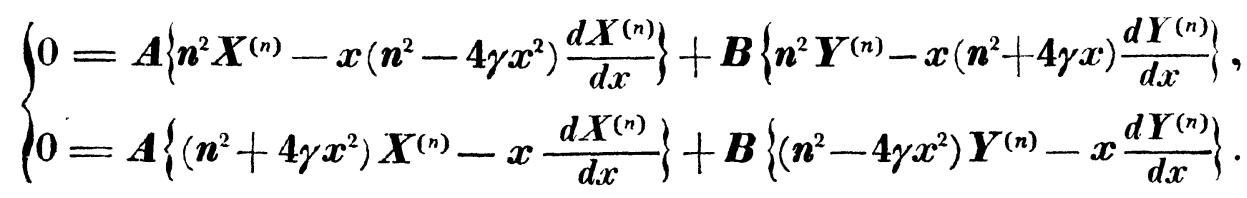

Diese Gleichungen sind zu erfüllen für $r=l$, d. h. für $x=\lambda l$. Hierzu ist nöthig, dafs ihre Determinante verschwinde, da $\boldsymbol{A}$ und $\boldsymbol{B}$ nicht $=0$ sein sollen. Es mufs also für $x=\lambda l$ :

$$
\text { (15.) } \begin{aligned}
& 0= 8 \gamma n^{2} x^{2} X^{(n)} Y^{(n)} \\
&-8 \gamma n^{2} x^{3}\left(X^{(n)} \frac{d Y^{(n)}}{d x}+Y^{(n)} \frac{d X^{(n)}}{d x}\right. \\
&-\left(n^{2}\left(n^{2}-1\right) x+16 \gamma^{2} x^{5}\right)\left(X^{(n)} \frac{d Y^{(n)}}{d x}-Y^{(n)} \frac{d X^{(n)}}{d x}\right) \\
&+8 \gamma \cdot x^{4} \frac{d X^{(n)}}{d x} \frac{d Y^{(n)}}{d x}
\end{aligned}
$$

sein. Bestimmt man aus dieser Gleichung $\lambda$ und aus einer der beiden Gleichungen (14.) das Verhältnifs von $\boldsymbol{A}$ und $\boldsymbol{B}$, so ist den Bedingungen (2.) genügt. Wir wollen eine Wurzel der Gleichung (15.) durch $\lambda_{n \mu}$ bezeichnen und

$$
\text { (16.) } \begin{aligned}
& U_{n \mu}= X^{(n)}\left\{\left(n^{2}-4 \gamma x^{2}\right) Y^{(n)}-x \frac{d Y^{(n)}}{d x}\right\} \\
&-Y^{(n)}\left\{\left(n^{2}+4 \gamma x^{2}\right) X^{(n)}-x \frac{d X^{(n)}}{d x}\right\} \\
& x=\lambda_{n \mu} l
\end{aligned}
$$

setzen; dann wird den Gleichungen (1. und 2.) durch

$$
w=C_{n \mu} \sin \left(4 \lambda_{n \mu}^{2} a t\right) \cos n \psi U_{n \mu},
$$

oder auch durch

$$
\text { (17.) } \begin{aligned}
\boldsymbol{w}= & \left\{\cos \left(4 \lambda_{n \mu}^{2} a t\right)\left(\boldsymbol{A}_{n \mu} \cos n \psi+\boldsymbol{B}_{n \mu} \sin n \psi\right)\right. \\
& \left.+\sin \left(4 \lambda_{n \mu}^{2} a t\right)\left(\boldsymbol{C}_{n \mu} \cos n \psi+\boldsymbol{D}_{n \mu} \sin n \psi\right)\right\} \boldsymbol{U}_{n \mu}
\end{aligned}
$$

genügt; wo $\boldsymbol{A}_{n \mu}, \boldsymbol{B}_{n \mu}, \boldsymbol{C}_{n \mu}, \boldsymbol{D}_{n \mu}$ willkürliche Constanten bezeichnen.

Wir wollen nun die Gleichung (15.) näher untersuchen und zuerst die Seite derselben rechts in eine Reihe entwickeln, die nach positiven Po- 
tenzen von $\boldsymbol{x}$ fortschreitet. Wir müssen zunächst das Product $\boldsymbol{X}^{(n)} \boldsymbol{Y}^{(n)}$ bilden. Hierbei läfst sich aber die directe Multiplication der unendlichen Reihen (10.) umgehen. Man kann nämlich durch Benutzung der Differentialgleichungen (9.), denen $\boldsymbol{X}^{(n)}$ und $\boldsymbol{Y}^{(n)}$ genügen, eine lineäre Differentialgleichung vierter Ordnung für $\boldsymbol{X}^{(n)} \boldsymbol{Y}^{(n)}$ finden und aus dieser das Product bestimmen, indem man auf die Form Rücksicht nimmt, die dasselbe augenscheinlich haben mufs. Man setze

$$
\boldsymbol{H}=\boldsymbol{X}^{(n)} \boldsymbol{Y}^{(n)}
$$

und bilde durch wiederholte Differentiation dieser Gleichung die Werthe der vier ersten Differentialquotienten von $\boldsymbol{H}$ nach $\boldsymbol{x}$. Dann drücke man die zweiten und höheren Differentialquotienten von $\boldsymbol{X}^{(n)}$ und $\boldsymbol{Y}^{(n)}$ mit Hülfe von (9.) durch diese Functionen selbst und ihre ersten Differentialquotienten aus. Dadurch erhält man fünf Gleichungen, welche $\boldsymbol{H}$ und dessen vier erste Differentialquotienten angeben, als lineare homogene Functionen der vier Gröfsen

$$
X^{(n)} Y^{(n)}, \quad X^{(n)} \frac{d Y^{(n)}}{d x}, \quad Y^{(n)} \frac{d X^{(n)}}{d x}, \quad \frac{d X^{(n)}}{d x} \frac{d Y^{(n)}}{d x} .
$$

Die erste dieser fünf Gleichungen multiplicire man mit (1.), die andern der Reihe nach mit den unbestimmten Coëfficienten $\boldsymbol{A}_{1}, \boldsymbol{A}_{2}, \boldsymbol{A}_{3}, \boldsymbol{A}_{4}$, addire alle und bestimme diese Coëfficienten so, dafs in der Summe die Factoren jener vier Gröfsen verschwinden; dann ergiebt sich identisch:

$$
0=H+A_{1} \frac{d H}{d x}+A_{2} \frac{d^{2} H}{d x^{2}}+A_{3} \frac{d^{3} H}{d x^{3}}+A_{4} \frac{d^{4} H}{d x^{4}} .
$$

Schreibt man die Gleichungen, durch welche die zweiten und höhern Differentialquotienten von $\boldsymbol{X}^{(n)}$ und $\boldsymbol{Y}^{(n)}$ durch diese Gröfsen selbst und deren erste Differentialquotienten ausgedrückt werden, folgendermaafsen:

$$
\begin{array}{ll}
\frac{d^{2} X^{(n)}}{d x^{2}}=a_{2} X^{(n)}+a_{2}{ }^{\prime} \frac{d X^{(n)}}{d x}, & \frac{d^{2} Y^{(n)}}{d x^{2}}=b_{2} Y^{(n)}+b_{2}{ }^{\prime} \frac{d Y^{(n)}}{d x}, \\
\frac{d^{3} X^{(n)}}{d x^{3}}=a_{3} X^{(n)}+a_{3}{ }^{\prime} \frac{d X^{(n)}}{d x}, & \frac{d^{3} Y^{(n)}}{d x^{3}}=b_{3} Y^{(n)}+b_{3}{ }^{\prime} \frac{d Y^{(n)}}{d x}, \\
\frac{d^{4} X^{(n)}}{d x^{4}}=a_{4} X^{(n)}+a_{4}{ }^{\prime} \frac{d X^{(n)}}{d x}, & \frac{d^{4} Y^{(n)}}{d x^{4}}=b_{4} Y^{(n)}+b_{4}{ }^{\prime} \frac{d Y^{(n)}}{d x},
\end{array}
$$

so ergeben sich für die Coëfficienten $\boldsymbol{A}$ die Gleichungen:

$$
\begin{aligned}
1+\left(a_{2}+b_{2}\right) A_{2}+\left(a_{3}+b_{3}\right) A_{3}+\left(a_{4}+b_{4}+6 a_{2} b_{2}\right) A_{4}=0 \\
A_{1}+\quad a_{2}^{\prime} A_{2}+\left(a_{3}^{\prime}+3 b_{2}\right) A_{3}+\left(a_{4}^{\prime}+4 b_{3}+6 a_{2}^{\prime} b_{2}\right) A_{4}=0, \\
A_{1}+\quad b_{2}^{\prime} A_{2}+\left(b_{3}^{\prime}+3 a_{2}\right) A_{3}+\left(b_{4}^{\prime}+4 a_{3}+6 a_{2} b_{2}^{\prime}\right) A_{4}=0 \\
2 A_{2}+3\left(a_{2}^{\prime}+b_{2}^{\prime}\right) A_{3}+\left(4 a_{3}^{\prime}+4 b_{3}^{\prime}+6 a_{2}^{\prime} b_{2}^{\prime}\right) A_{4}=0 .
\end{aligned}
$$

Substituirt man in diese Gleichungen für die Gröfsen $a, b$ ihre Werthe, die 
76 4. Kirchhoff, ̈̈b.d. Gleichgewicht u. d. Bewegung einer elastischen Scheibe.

sich aus (9.) ergeben, löset dieselben auf und setzt die Werthe von $A_{1}, A_{2}$, $\boldsymbol{A}_{3}, \boldsymbol{A}_{4}$, die man findet, in die Gleichung (18.), so wird diese zu:

$$
0=64 H+\frac{4 n^{2}-1}{x^{3}} \frac{d H}{d x}-\frac{4 n^{2}-1}{x^{2}} \frac{d^{2} H}{d x^{2}}+\frac{4}{x} \frac{d^{3} H}{d x^{3}}+\frac{d^{4} H}{d x^{4}} .
$$

Aus (10.) folgt aber, dafs

$$
\text { (19.) } H=X^{(n)} Y^{(n)}=\frac{x^{2 n}}{(1.2 .3 \ldots n)^{2}}\left(1+B_{1} x^{4}+B_{2} x^{8}+B_{3} x^{12}+B_{4} x^{16}+\ldots\right)
$$

sein mufs; substituirt man diese Reihe in die eben gefundene Differentialgleichung, so findet sich

also

$$
\boldsymbol{B}_{k}=-\frac{\boldsymbol{B}_{k-1}}{k \cdot n+k \cdot n+2 k-1 \cdot n+2 k}
$$

(20.) $\quad \boldsymbol{B}_{k}=\frac{(-1)^{k}}{1.2 \ldots k . n+1 . n+2 \ldots n+k . n+1 . n+2 \ldots n+2 k}$.

Um die Gleichung (15.) zu bilden, sind ferner die Ausdrücke:

$$
X^{(n)} \frac{d Y^{(n)}}{d x}+Y^{(n)} \frac{d X^{(n)}}{d x}, \quad X^{(n)} \frac{d Y^{(n)}}{d x}-Y^{(n)} \frac{d X^{(n)}}{d x}, \quad \frac{d X^{(n)}}{d x} \frac{d Y^{(n)}}{d x}
$$

zu entwickeln. Wir benutzen hierzu die folgenden Gleichungen, die identisch sind in Rücksicht auf die Gleichungen (9.):

$$
\begin{array}{rc}
H= & X^{(n)} Y^{(n)}, \\
\frac{d H}{d x}= & X^{(n)} \frac{d Y^{(n)}}{d x}+Y^{(n)} \frac{d X^{(n)}}{d x} \\
\frac{d^{2} H}{d x^{2}}= & \frac{2 n^{2}}{x^{2}} X^{(n)} Y^{(n)}-\frac{1}{x}\left(X^{(n)} \frac{d Y^{(n)}}{d x}+Y^{(n)} \frac{d X^{(n)}}{d x}\right)+2 \frac{d X^{(n)}}{d x} \frac{d Y^{(n)}}{d x} \\
\frac{d^{3} H}{d x^{3}}=-\frac{6 n^{2}}{x^{3}} X^{(n)} Y^{(n)}+\frac{4 n^{2}+2}{x^{2}}\left(X^{(n)} \frac{d Y^{(n)}}{d x}+Y^{(n)} \frac{d X^{(n)}}{d x}\right) \\
+8\left(X^{(n)} \frac{d Y^{(n)}}{d x}-Y^{(n)} \frac{d X^{(n)}}{d x}\right)-\frac{6}{x} \frac{d X^{(n)}}{d x} \frac{d Y^{(n)}}{d x} .
\end{array}
$$

Durch Auflősung dieser Gleichungen erhält man

$$
\begin{aligned}
X^{(n)} \frac{d Y^{(n)}}{d x}+Y^{(n)} \frac{d X^{(n)}}{d x} & =\frac{d H}{d x}, \\
X^{(n)} \frac{d Y^{(n)}}{d x}-Y^{(n)} \frac{d X^{(n)}}{d x} & =\frac{d^{3} H}{d x^{3}}+\frac{3}{x} \frac{d^{2} H}{d x^{2}}-\frac{4 n^{2}-1}{x^{2}} \frac{d H}{d x}, \\
\therefore \quad \frac{d X^{(n)}}{d x} \frac{d Y^{(n)}}{d x} & =\frac{d^{2} H}{d x^{2}}+\frac{1}{x} \frac{d H}{d x}-\frac{2 n^{2}}{x^{2}} H .
\end{aligned}
$$

Durch Berücksichtigung der Gleichungen (19. und 20.) werden diese drei Grofsen der Reihe nach: 


$$
\begin{aligned}
& \frac{2 x^{2 n-1}}{(1.2 \ldots n)^{2}}\left(n+\sum_{1}^{\infty} k(n+2 k) \boldsymbol{B}_{k} x^{4 k}\right), \\
& \frac{4 x^{2 n-3}}{(1.2 \ldots n)^{2}} \sum_{1}^{\infty} k(n+k)(n+2 k) B_{k} x^{4 k}, \\
& \frac{x^{2 n-2}}{(1.2 \ldots n)^{2}}\left(n^{2}+\sum_{1}^{\infty} k\left(2(n+2 k)^{2}-n^{2}\right) B_{k} x^{4 k}\right) .
\end{aligned}
$$

Hiernach wird die Gleichung (15.), wenn man den Factor $x^{2 n+2}$ wegläfst, folgende:

$$
0=(4 \gamma-1) n^{2}(n-1)+\sum_{1}^{\infty} k\left(-1^{k}\right) \frac{E_{k}}{M_{k}} x^{4 k},
$$

wo

$$
\begin{aligned}
& \boldsymbol{E}_{k}=-n^{2}\left(n^{2}-1\right)+4 \gamma(n+2 k)(n+2 k+1)(n(n-1)-2 k+4 \gamma k(n+k)), \\
& \boldsymbol{M}_{k}=1.2 \ldots k \cdot n+1 . n+2 \ldots n+k \cdot n+1 . n+2 \ldots n+2 k+1 .
\end{aligned}
$$

Man sieht, dafs die Gleichung (21.), deren Wurzeln, durch $l$ dividirt, die Werthe sind, die für $\lambda_{n \mu}$ gesetzt werden können, nur die Potenzen von $x$ enthält, deren Exponenten Vielfache von 4 sind. Es folgt daraus, dafs, wenn $l \lambda$ eine ihrer Wurzeln ist, auch $-l \lambda, l \lambda \sqrt{ }-1$ und $-l \lambda \sqrt{ }-1$ ihre Wurzeln sind. Ich will jetzt nachweisen, dafs die vierten Potenzen aller Wurzeln der Gleichung (21.) reell und positiv sind; daraus wird dann hervorgehen, dafs unter jeder Gruppe von vier Wurzeln, wie die angegebene deren eine ist, sich eine reelle positive Wurzel befinden mufs. Wie schon früher bemerkt, ist $\theta$ eine positive Gröfse; es ist also $\gamma$, welches $=\frac{1+2 \theta}{1+\theta}$ gesetzt wurde, gröfser als 1. Hieraus folgt, dafs $\boldsymbol{E}_{k}$ immer positiv ist, dafs also die Glieder der rechten Seite der Gleichung (21.) abwechselnde Zeichen haben. Hiernach ist es unmöglich, dafs die vierte Potenz einer Wurzel dieser Gleichung negativ sei. Dafs sie auch nicht imaginär sein kann, läfst sich auf folgende, indirecte Weise darthun.

Es seien $l \lambda$ und $l \lambda^{\prime}$ zwei Wurzeln der Gleichung (21.). Für die erste derselben sei die durch (16.) bestimmte Function $U_{n \mu}=U$, für die zweite $=U^{\prime}:$ dann läfst sich zeigen, dafs

$$
\text { (22.) }\left(\lambda^{4}-\lambda^{\prime 4}\right) \int_{0}^{l} U U^{\prime} r d r=0
$$

ist. Wir wollen es als bewiesen annehmen und wollen zeigen, dafs dann $\lambda^{\prime \prime}$ nicht imaginär sein kann. Es sei

$$
\begin{aligned}
\lambda & =\boldsymbol{p}+q \sqrt{ }-1 \\
\boldsymbol{U} & =\boldsymbol{p}+\boldsymbol{Q} \sqrt{ }-1
\end{aligned}
$$


Eine andere Wurzel der Gleichung (21.) mufs dann $(p-q \sqrt{ }-1) l$ sein. Wir setzen

$$
\lambda^{\prime}=p-q \sqrt{ }-1 \text { : }
$$

dann wird

Die Gleichung (22.) wird dann:

$$
U^{\prime}=\boldsymbol{P}-\boldsymbol{Q}_{\sqrt{ }-1}
$$

$$
p q\left(p^{2}-q^{2}\right) \int_{0}^{l}\left(\boldsymbol{P}^{2}+Q^{2}\right) \boldsymbol{r} d \boldsymbol{r}=0
$$

Das hier vorkommende Integral kann nicht verschwinden, da es eine Summe von lauter positiven Gröfsen ist, also mufs

$$
p q\left(p^{2}-q^{2}\right)=0
$$

sein; und dieses ist die Bedingung dafür, dafs $\lambda^{4}$ reell ist.

Wir wollen nun die Richtigkeit der Gleichung (22.) erweisen. Daraus, dafs der in (17.) gegebene Ausdruck für $w$ der Gleichung (1.) genügt, folgt, dafs, wenn man

$$
\frac{d^{2} U}{d r^{2}}+\frac{1}{r} \frac{d U}{d r}-\frac{n^{2}}{r^{2}} U=4 \lambda^{2} V
$$

setzt, gleichzeitig

$$
\left\{\begin{array}{l}
\frac{d^{2} U}{d r^{2}}+\frac{1}{r} \frac{d U}{d r}-\frac{n^{2}}{r^{2}} U=4 \lambda^{2} V, \\
\frac{d^{2} V}{d r^{2}}+\frac{1}{r} \frac{d V}{d r}-\frac{n^{2}}{r^{2}} V=4 \lambda^{2} U
\end{array}\right.
$$

ist. Daraus, dafs derselbe Ausdruck für $w$ die Gleichungen (2.) oder die Gleichungen (13.), welche dieselben sind, erfüllt, folgt, dafs für $r=l$,

$$
\left\{\begin{array}{c}
\frac{1+2 \theta}{1+\theta} \frac{d}{d r}\left(\frac{d^{2} U}{d r^{2}}+\frac{1}{r} \frac{d U}{d r}-\frac{n^{2}}{r^{2}} U\right)-\frac{n^{2}}{r^{2}}\left(\frac{d U}{d r}-\frac{1}{r} U\right)=0, \\
\frac{\theta}{1+\theta}\left(\frac{d^{2} U}{d r^{2}}+\frac{1}{r} \frac{d U}{d r}-\frac{n^{2}}{r^{2}} U\right)+\frac{d^{2} U}{d r^{2}}=0
\end{array}\right.
$$

sein wird. Die Gleichungen (23.) lassen sich wie folgt schreiben:

$$
\begin{aligned}
4 \lambda^{2} V & =r^{n-1} \frac{d}{d r} \frac{1}{r^{2 n-1}} \frac{d}{d r} r^{n} U, \\
4 \lambda^{2} U & =r^{n-1} \frac{d}{d r} \frac{1}{r^{2 n-1}} \frac{d}{d r} r^{n} V .
\end{aligned}
$$

Durch Substitution des Werths von $\boldsymbol{V}$ aus der ersten dieser beiden Gleichungen in die zweite erhält man

$$
16 \lambda^{4} U=r^{n-1} \frac{d}{d r} \frac{1}{r^{2 n-1}} \frac{d}{d r} r^{2 n-1} \frac{d}{d r} \frac{1}{r^{2 n-1}} \frac{d}{d r} r^{n} U
$$

Auf dieselbe Weise ergiebt sich eine Gleichung, die man aus dieser erhält, wenn man $U^{\prime}$ und $\lambda^{\prime}$ statt $U$ und $\lambda$ setzt. Substituirt man den Werth von 
$16 \lambda^{4} U$ aus (25.) in das Integral

$$
16 \lambda^{4} \int U U^{\prime} r d r
$$

und integrirt viermal partiell, so kommt man auf das Integral

und dieses ist

$$
\int \boldsymbol{r} d r \boldsymbol{U} \cdot \boldsymbol{r}^{n-1} \frac{d}{d r} \frac{1}{r^{2 n-1}} \frac{d}{d r} r^{2 n-1} \frac{d}{d r} \frac{1}{r^{2 n-1}} \frac{d}{d r} \boldsymbol{r}^{n} \boldsymbol{U}^{\prime}
$$

$$
=16 \lambda^{\prime 4} \int \boldsymbol{U} \boldsymbol{U}^{\prime} r d r \text {. }
$$

Man erhält auf dem angedeuteten Wege die Gleichung

$$
\begin{aligned}
\left.16 \lambda^{4}-\lambda^{\prime 4}\right) \int \boldsymbol{U} \boldsymbol{U}^{\prime} \boldsymbol{r} d \boldsymbol{r}= & \frac{1}{r^{n-1}} \boldsymbol{U}^{\prime}\left(\frac{d}{d r} \boldsymbol{r}^{2 n-1} \frac{d}{d r} \frac{1}{r^{2 n-1}} \frac{d}{d r} \boldsymbol{r}^{n} \boldsymbol{U}\right) \\
& -\left(\frac{d}{d r} \boldsymbol{r}^{n} U^{\prime}\right)\left(\frac{d}{d r} \frac{1}{r^{2 n-1}} \frac{d}{d r} \boldsymbol{r}^{n} \boldsymbol{U}\right) \\
& +\left(\frac{d}{d r} \frac{1}{r^{2 n-1}} \frac{d}{d r} r^{n} U^{\prime}\right)\left(\frac{d}{d r} r^{n} U\right) \\
& -\left(\frac{d}{d r} r^{2 n-1} \frac{d}{d r} \frac{1}{r^{2 n-1}} \frac{d}{d r} r^{n} U^{\prime}\right) \frac{1}{r^{n-1}} U
\end{aligned}
$$

Nun läfst sich zeigen, dafs der Ausdruck auf der rechten Seite dieser Gleichung sowohl für $r=0$, als für $r=l$ verschwindet. Dafs er für $r=0$ verschwindet, ist einzusehen, wenn man erwägt, dafs $\boldsymbol{U}$ und $\boldsymbol{U}^{\prime}$ von der Form

$$
\boldsymbol{c} \boldsymbol{r}^{n}+\boldsymbol{c}_{1} \boldsymbol{r}^{n+2}+\boldsymbol{c}_{2} \boldsymbol{r}^{n+4}+\ldots
$$

sind. Um zu beweisen, dafs er für $r=l$ verschwindet, wenden wir die Gleichungen (24.) an. Diese lassen sich folgendermaafsen schreiben:

$$
\begin{aligned}
& \frac{d^{2} U}{d r^{2}}=\frac{\theta}{1+2 \theta}\left(\frac{n^{2}}{r^{2}} U-\frac{1}{r} \frac{d U}{d r}\right) . \\
& \frac{d^{3} U}{d r^{3}}=-\frac{3 n^{2}}{r^{3}} C+\frac{n^{2}+\left(n^{2}+1\right)(1+3 \theta)}{(1+2 \theta) r^{2}} \frac{d U}{d r} .
\end{aligned}
$$

Mit Hülfe dieser Gleichungen und derer, welche aus ihnen entstehen, wenn man $\boldsymbol{U}^{\prime}$ statt $\boldsymbol{U}$ setzt, drücke man die zweiten und dritten Differentialquotienten von $U$ und $U^{\prime}$, auf welche man kommt, wenn man die in (26.) angegebenen Differentiationen ausführt, durch $U$ und $U^{\prime}$ selbst und ihre ersten Differentialquotienten aus; man findet dann, dafs die Glieder auf der rechten Seite von (26.) sich gegenseitig aufheben. Es folgt daraus die Richtigkeit der Gleichung (22.).

Es ist bis jetzt auf die Bedingungen nicht Rücksicht genommen worden, welche die Lösung unserer partiellen Differentialgleichung für $t=0$ erfüllen soll. Wir werden nun die gefundene Lösung (17.) so zu verallgemeinern suchen, dafs auch diesen Bedingungen genügt werden kann. Von dem Ausdrucke, 
80 4. Kirchhoff, üb. d. Gleichgewicht u. d. Bewegung einer elastischen Scheibe.

der in (17.) $=w$ gesetzt ist, kann man die Summe in Beziehung auf $\mu$ und in Beziehung auf $n$ nehmen, und diese Doppelsumme $=w$ setzen. Bei der ersten der beiden Summationen kann man sich darauf beschränken, die reellen positiven Werthe $\lambda_{n \mu}$ zu berücksichtigen; durch die Berücksichtigung der negativen und imaginären Werthe $\lambda_{n \mu}$ gewinnt man nichts an Allgemeinheit des Ausdrucks von $w$; denn: ist wiederum

$$
\boldsymbol{U}_{n \mu}=\boldsymbol{U} \text { für } \lambda_{n \mu}=\lambda
$$

und

$$
\boldsymbol{U}_{n \mu}=\boldsymbol{U}^{\prime} \text { für } \lambda_{n \mu}=\lambda^{\prime} \text {, }
$$

so ist für denselben Werth von $r$ :

$$
\boldsymbol{U}=\boldsymbol{U}^{\prime}, \quad \text { falls } \lambda=-\lambda^{\prime}
$$

und

Wir wollen jetzt unter

$$
\boldsymbol{U}=(-1)^{n+1} \boldsymbol{U}^{\prime}, \text { falls } \lambda=\lambda^{\prime} \gamma^{\prime}-1
$$

$$
l \lambda_{n_{0}}, l \lambda_{n_{1}}, l \lambda_{n_{2}}, \ldots l \lambda_{n_{\mu}}, \ldots
$$

die positiven reellen Wurzeln der Gleichung (21.) verstehen, dieselben ihrer Gröfse nach so geordnet, dafs $l \lambda_{n_{0}}$ die kleinste ist, und wollen

$$
\begin{aligned}
\boldsymbol{w}=\sum_{0}^{\infty} \sum_{0}^{\infty} \mu & \left\{\cos \left(4 \lambda_{n \mu}^{2} a t\right)\left(\boldsymbol{A}_{n \mu} \cos n \psi+\boldsymbol{B}_{n \mu} \sin n \psi\right)\right. \\
& \left.+\sin \left(4 \lambda_{n \mu}^{2} a t\right)\left(\boldsymbol{C}_{n \mu} \cos n \psi+\boldsymbol{D}_{n \mu} \sin n \psi\right)\right\} \boldsymbol{U}_{n \mu}
\end{aligned}
$$

setzen. Die Constanten $\boldsymbol{A}, \boldsymbol{B}, \boldsymbol{C}, \boldsymbol{D}$ müssen nun so bestimmt werden, dafs für $\boldsymbol{t}=\mathbf{0}, \quad \boldsymbol{v}=\boldsymbol{F}(\boldsymbol{r}, \psi)$,

$$
\frac{\partial w}{\partial t}=\boldsymbol{\Phi}(\boldsymbol{r}, \psi)
$$

wird, wo $\boldsymbol{F}$ und $\Phi$ zwei gegebene Functionen von $\boldsymbol{r}$ und $\psi$ bedeuten. Aus der ersten Bedingung werden sich die Werthe der Gröfsen $\boldsymbol{A}, \boldsymbol{B}$ ergeben, aus der zweiten die Werthe der Gröfsen $C, D$; und zwar diese auf ganz ähnliche Weise, als jene; es ist also hinreichend, zu zeigen, wie jene gefunden werden können. Die erste Bedingung erfordert, dafs

$$
\boldsymbol{F}(\boldsymbol{r}, \psi)=\sum_{0}^{\infty} \sum_{0}^{\infty} \mu\left(\boldsymbol{A}_{n \mu} \cos n \psi+\boldsymbol{B}_{n \mu} \sin n \psi\right) \boldsymbol{U}_{n \mu}
$$

werde. Wir stellen uns $\boldsymbol{F}(\boldsymbol{r}, \psi)$ nach den Cosinus und Sinus der Vielfachen von $\psi$ entwickelt vor, so dafs

$$
\begin{aligned}
\boldsymbol{F}(\boldsymbol{r}, \psi)=\boldsymbol{F}_{0}(\boldsymbol{r}) & +\boldsymbol{F}_{1}(\boldsymbol{r}) \cos \psi+\boldsymbol{F}_{2}(\boldsymbol{r}) \cos 2 \psi+\ldots \\
& +\boldsymbol{F}_{1}^{\prime}(\boldsymbol{r}) \sin \psi+\boldsymbol{F}_{2}^{\prime}(\boldsymbol{r}) \sin 2 \psi+\ldots
\end{aligned}
$$

ist, und dann diese Entwickelung für $\boldsymbol{F}(\boldsymbol{r}, \psi)$ substituirt. Dann zeigt sich, dafs 
4. Kirchhoff, üb. d. Gleichgewicht u.d. Bewegung einer elastischen Scheibe. 81

$$
\left\{\begin{array}{l}
\boldsymbol{F}_{n}(\boldsymbol{r})=\sum_{\mathbf{0}}^{\infty} \boldsymbol{A} \boldsymbol{A}_{n \mu} \boldsymbol{U}_{n \mu}, \\
\boldsymbol{F}_{n}^{\prime}(\boldsymbol{r})=\sum_{\mathbf{0}}^{\infty} \mu \boldsymbol{B}_{n \mu} \boldsymbol{U}_{n \mu}
\end{array}\right.
$$

sein mufs. Die Functionen $\boldsymbol{F}_{n}(\boldsymbol{r})$ und $\boldsymbol{F}_{n}^{\prime}(\boldsymbol{r})$ lassen sich als gegeben betrachten; die Bestimmung $\operatorname{der}$ Gröfsen $\boldsymbol{A}$ und $\boldsymbol{B}$ ist also auf die Aufgabe reducirt, eine gegebene Function von $\boldsymbol{r}$ nach den Functionen $\boldsymbol{U}_{n_{0}}, \boldsymbol{U}_{n_{1}}, \boldsymbol{U}_{n_{2}}, \ldots$ zu entwickeln. Vorausgesetzt, dafs diese Entwicklung möglich ist, kann man die Coëfficienten derselben mit Hülfe des Satzes finden, der durch die Gleichung (22.) ausgesprochen wird. Diese Gleichung zeigt, dafs, wenn $\mu$ und $\mu^{\prime}$ zwei verschiedene Zahlen sind,

$$
\int_{0}^{l l} U_{n \mu} U_{n \mu^{\prime}} \boldsymbol{r} d \boldsymbol{r}=0
$$

ist; es folgt daher aus den Gleichungen (27.):

$$
\begin{aligned}
& \boldsymbol{A}_{n \mu} \int_{\mathbf{0}}^{l} U_{n \mu} U_{n \mu} \boldsymbol{r} d \boldsymbol{r}=\int_{\mathbf{0}}^{l} \boldsymbol{F}_{n}(\boldsymbol{r}) \boldsymbol{U}_{n \mu} \boldsymbol{r} d \boldsymbol{r}, \\
& \boldsymbol{B}_{n \mu} \int_{\mathbf{0}}^{l} \boldsymbol{U}_{n \mu} \boldsymbol{U}_{n \mu} \boldsymbol{r} d \boldsymbol{r}=\int_{\mathbf{0}}^{l} \boldsymbol{F}_{n}^{\prime}(\boldsymbol{r}) \boldsymbol{U}_{n \mu} \boldsymbol{r} d \boldsymbol{r} .
\end{aligned}
$$

\$. 5.

Für die Vergleichung der Theorie mit der Erfahrung ist die Untersuchung des Falles wichtig, in welchem die Schwingungen der Scheibe, von der Art sind, dafs sie einen reinen Ton erzeugen. Die Verhältnisse der Schwingungszahlen der verschiedenen Töne, welche eine Scheibe geben kann, und die Knotenlinien, die bei jedem einzelnen vorhanden sind, bieten sich hier als die hauptsächlichsten Vergleichungspuncte dar. Mit diesem Falle wollen wir uns jetzt beschäftigen. In demselben mufs $w$ durch folgenden Ausdruck dargestellt sein :

$$
\text { (28.) } \begin{aligned}
\boldsymbol{w}= & \left\{\cos \left(4 \lambda_{n \mu}^{2} \boldsymbol{a t}\right)(\boldsymbol{A} \cos n \psi+\boldsymbol{B} \sin n \psi)\right. \\
& \left.+\sin 4 \lambda_{n \mu}^{2} \boldsymbol{a t}(\boldsymbol{C} \cos n \psi+\boldsymbol{D} \sin n \psi)\right\} \boldsymbol{U}_{n \mu} .
\end{aligned}
$$

Der Ton ist durch $\lambda_{n \mu}$ in der Art bestimmt, dafs seine Schwingungszahl, d. h. die Anzahl der Schwingungen, die in der Einheit der Zeit vollführt werden,

$$
=\frac{4 \lambda_{n \mu}^{2} a}{\pi}
$$

ist. Die Knotenlinien sind diejenigen. Linien, für welche, für alle Werthe von $t, w=0$ ist; sie werden daher die Puncte enthalten, für welche entweder die Gleichung

$$
\text { (29.) } \boldsymbol{U}_{n \mu}=0
$$

Crelle's Journal f. d. M. Bd. XL. Heft 1. 
erfüllt wird, oder die beiden Gleichungen

$$
\text { (30.) }\left\{\begin{array}{l}
\boldsymbol{A} \cos n \psi+\boldsymbol{B} \sin n \psi=0 \\
\boldsymbol{C} \cos n \psi+\boldsymbol{D} \sin n \psi=0
\end{array}\right.
$$

bestehen. Die Gleichung (29.) liefert gewisse Werthe von $\boldsymbol{r}$ als Wurzeln. So viele reelle Wurzeln sie hat, die kleiner als $l$ sind, so viele, mit der Peripherie der Scheibe concentrische Kreise werden in der Klangfigur vorkommen; die Anzahl und Gröfse derselben wird allein von dem Tone abhangen und unabhängig sein von den Werthen der Coëfficienten $\boldsymbol{A}, \boldsymbol{B}, \boldsymbol{C}, \boldsymbol{D}$. Die Gleichungen (30.) werden für keinen Punct erfüllt, wenn nicht

$$
\boldsymbol{A}: \boldsymbol{B}=\boldsymbol{C}: \boldsymbol{D}
$$

ist; in diesem Falle sind jene Kreise die einzigen Knotenlinien. Besteht diese Proportion, so geben die Gleichungen (30.) $\boldsymbol{n}$ Werthe von $\psi$, von denen je zwei aufeinanderfolgende um $\frac{\pi}{n}$ unterschieden sind; dann kommen also zu jenen Kreisen noch $\boldsymbol{n}$ Durchmesser als Knotenlinien hinzu, welche die Peripherie der Scheibe, in gleiche Theile theilen.

Diese allgemeinen Resultate der Theorie sind im Wesentlichen mit der Erfahrung in Übereinstimmung. Der Versuch zeigt, dafs die Knotenlinien aus Kreisen bestehen, die mit der Peripherie der Scheibe concentrisch sind, und aus Durchmessern, die diese in gleiche Theile theilen, wenn man von gewissen Verzerrungen absieht, die diese Linien erleiden und die, wie mir scheint, hauptsächlich darin ihren Grund haben, dafs die Scheibe nicht vollkommen frei ist, wie die Theorie sie voraussetzt. Der Versuch zeigt aber auch, dafs bei einem Tone, bei dem zuweilen Durchmesser als Knotenlinien vorkommen, die Durchmesser zuweilen fehlen. Fehlen sie, so ordnet sich der auf die Scheibe gestreute Sand zwar auch in Durchmessern an: diese bleiben aber nicht fest während der Bewegung der Scheibe, sondern oscilliren. Wollte man diese interessante Erscheinung zu erklären versuchen, so müfste man die Bewegung eines Sandkornes verfolgen, welches, von einer Stelle der Scheibe fortgeschnellt, auf eine andere fällt, und so von einer Stelle zur anderen geschleudert wird, während die Scheibe selbst die durch die Gleichung (28.) ausgedrückte Bewegung vollführt. Auf diese Betrachtung will ich indessen hier nicht näher eingehen.

Chladni hat durch Versuche gefunden, dafs die Schwingungszahlen der Töne, die in ihren Klangfiguren dieselbe Anzahl von Durchmessern haben, (d. h. der Töne, die demselben Werthe von $n$ entsprechen), mit Ausnahme 
der tiefsten, sich nahe wie die Quadrate aufeinanderfolgender, gerader oder ungerader Zahlen verhalten, je nachdem die Zahl der Durchmesser gerade oder ungerade ist. Ich will jetzt nachweisen, dafs die Theorie dasselbe Gesetz liefert. Es geht dies aus einer Umformung der Gleichung (21.) hervor.

Für die Function $\boldsymbol{Y}^{(0)}$, die durch die zweite der Gleichungen (10.) bestimmt ist, hat Poisson folgende semiconvergente Reihe entwickelt:

$$
\begin{aligned}
Y^{(0)}= & \frac{1}{\sqrt{ } 2} \pi \frac{1}{\sqrt{ } x}\left\{(\cos 2 x+\sin 2 x)\left(1-\frac{(1.3)^{2}}{1.2} \frac{1}{(16 x)^{2}}+\frac{(1.3 .5 .7)^{2}}{1.2 .3 .4} \frac{1}{(16 x)^{4}}-\ldots\right)\right\} \\
& \left.+(\sin 2 x-\cos 2 x)\left(\frac{1^{2}}{1} \frac{1}{16 x}-\frac{(1.3 .5)^{2}}{1.2 .3} \frac{1}{(16 x)^{3}}+\frac{(1.3 .5 .7 .9)^{2}}{1.2 .3 .4 .5} \frac{1}{(16 x)^{5}}-\ldots\right)\right\} .
\end{aligned}
$$

Auf eine ähnliche Weise findet man

$$
X^{(1)}=\frac{1}{2 \sqrt{ } \pi} \frac{e^{2 x}}{\sqrt{ } x}\left\{1+\frac{1^{2}}{1} \frac{1}{16 x}+\frac{(1.3)^{2}}{1.2} \frac{1}{(16 x)^{2}}+\frac{(1.3 .5)^{2}}{1.2 .3} \frac{1}{(16 x)^{3}}+\ldots\right\} .
$$

Nun folgt aus den Gleichungen (10.)

$$
\begin{aligned}
& Y^{(n+1)}=-\frac{1}{2} x^{n} \frac{d}{d x}\left(\frac{Y^{(n)}}{x^{(n)}}\right) \\
& X^{(n+1)}=\frac{1}{2} x^{n} \frac{d}{d x}\left(\frac{X^{(n)}}{x^{n}}\right) .
\end{aligned}
$$

Setzt man hier $n=0$ und substituirt für $Y^{(0)}, Y^{(0)}$ die eben angegebenen Reihen, so erhält man ähnliche Reihen für $Y^{(1)}, X^{(1)}$; aus diesen findet man wiederum ähnliche Reihen für $\boldsymbol{Y}^{(2)}, \boldsymbol{X}^{(2)}$, u. s. f. Es ergiebt sich:

$$
\begin{aligned}
& Y^{(n)}=\frac{1}{\sqrt{2 \pi}} \frac{1}{\sqrt{x}}\left\{( \operatorname { c o s } ( 2 x - \frac { 1 } { 2 } n \pi ) + \operatorname { s i n } ( 2 x - \frac { 1 } { 2 } n \pi ) ) \left(1-\frac{\left(1-4 n^{2}\right)\left(9-4 n^{2}\right)}{1.2} \frac{1}{(16 x)^{2}}\right.\right. \\
& \left.+\frac{\left(1-4 n^{2}\right)\left(9-4 n^{2}\right)\left(25-4 n^{2}\right)\left(49-4 n^{2}\right)}{1.2 .3 .4} \frac{1}{(16 x)^{4}}+\ldots\right) \\
& +\left(\sin \left(2 x-\frac{1}{2} n \pi\right)-\cos \left(2 x-\frac{1}{2} n \pi\right)\right)\left(\frac{\left(1-4 n^{2}\right)}{1} \frac{1}{16 x}\right. \\
& \left.\left.-\frac{\left.1-4 n^{2}\right)\left(9-4 n^{2}\right)\left(25-4 n^{2}\right)}{1.2 .3} \frac{1}{(16 x)^{3}}+\ldots\right)\right\} \text {, } \\
& X^{(n)}=\frac{1}{2 \sqrt{ } \pi} \frac{e^{2 x}}{\sqrt{x}}\left\{1+\frac{\left(1-4 n^{2}\right)}{1} \frac{1}{16 x}+\frac{\left(1-4 n^{2}\right)\left(9-4 n^{2}\right)}{1.2} \frac{1}{(16 x)^{2}}\right. \\
& \left.+\frac{\left(1-4 n^{2}\right)\left(9-4 n^{2}\right)\left(25-4 n^{2}\right)}{1.2 .3} \frac{1}{(16 x)^{3}}+\ldots\right\} \text {. }
\end{aligned}
$$

Die erste dieser beiden Reihen ist in einer ein wenig andern Form schon von Herrn Professor Jacobi in Schumachers astronomischen Nachrichten Bd. 28, S. 94 angegeben. Substituirt man diese Werthe von $Y^{(n)}$ und $X^{(n)}$ in die Gleichung (15.), welche identisch mit der Gleichung (21.) ist, so kann 
man aus der resultirenden Gleichung $\log \left(2 x-\frac{1}{2} n \pi\right)$ ausdrücken als den Quotienten zweier Reihen, die nach den Potenzen von $\frac{1}{16 x}$ fortschreiten. Es ergiebt sich

$$
\text { (31.) } \operatorname{tang}\left(2 x-\frac{1}{2} n \pi\right)=\frac{\frac{\mathfrak{B}}{16 x}+\frac{\mathfrak{S}}{(16 x)^{2}}-\frac{\mathfrak{D}}{(16 x)^{3}}+\ldots}{\mathfrak{A}+\frac{\mathfrak{B}}{16 x}+\frac{\mathfrak{D}}{(16 x)^{3}}+\ldots} \text {, }
$$

wo

$$
\begin{aligned}
& \mathfrak{A}=\gamma \\
& \mathfrak{B}=\gamma\left(1-4 n^{2}\right)-8, \\
& \mathfrak{C}=\gamma\left(1-4 n^{2}\right)\left(9-4 n^{2}\right)+48\left(1+4 n^{2}\right), \\
& \mathfrak{D}=-\gamma \frac{1}{3}\left(\left(1-4 n^{2}\right)\left(9-4 n^{2}\right)\left(13-4 n^{2}\right)\right)+8\left(9+136 n^{2}+80 n^{4}\right) .
\end{aligned}
$$

Ist $\boldsymbol{x}$ grofs, so kann man die Seite rechts der Gleichung (31.) $=0$ setzen; dadurch erhält man, als Näherungswerthe der Wurzeln derselben, die Werthe, welche der Ausdruck

$$
\frac{1}{4} \pi(n+2 h)
$$

annimmt, wenn für $h$ ganze Zahlen gesetzt werden. Es folgt hieraus das oben ausgesprochene, von Chladni gefundene Gesetz, da die Schwingungszahlen der Töne den Quadraten der Wurzeln der Gleichung (31.) proportional sind. Diese Gleichung zeigt ferner, dafs die Proportionalität der Schwingungszahlen mit den Quadraten aufeinanderfolgender gerader oder ungerader Zahlen um so näher Statt finden wird, je höher die Töne sind; und sie liefert ein Mittel, auf eine bequeme Weise alle Töne, die zu einem Werthe von $n$ gehören, mit Ausnahme der tiefsten, mit grofser Genauigkeit zu bestimmen. Was die Zahl $h$ in dem Näherungswerthe von $l \lambda_{n \mu}$ anbetrifft, so zeigt die numerische Rechnung, dafs sie $=\mu$ ist; so dafs also für grofse Werthe von $\mu l \lambda_{n \mu}$ nahezu $=\frac{1}{4} \pi(n+2 \mu)$ ist: ein Resultat, welches in vollkommner Übereinstimmung mit den Beobachtungen von Chladni ist.

Um die tiefsten Töne für einen Werth von $n$ zu ermitteln, mufs man die kleinsten Wurzeln der Gleichung (21.) berechnen. Diese Gleichung kann man auf die Form

$$
0=1-\frac{x^{4}}{A_{1}}+\frac{x^{8}}{A_{2}}-\frac{x^{12}}{A^{3}}+\text { etc. },
$$

bringen, indem man sie für $n=0$ und $n=1$ durch $x^{4}$ dividirt. Die Rechnung giebt folgende Werthe von $\log A_{1}, \log A_{2}, \ldots$ : 
4. Kirchhoff, üb. d. Gleichgewicht u. d. Beweguny einer elastischen Scheibe. 85

für $\theta=\frac{1}{2}$, also $\gamma=\frac{4}{3}$ (nach Poisson):

$$
n=0 \quad n=1 \quad n=2 \quad n=3
$$

\begin{tabular}{|c|c|c|c|c|}
\hline $\log A_{1}$ & 0,6642079 & 1,3480266 & 0,2650703 & 0,9733073 \\
\hline $\log A_{2}$ & 2,5351132 & 3,5880591 & 2,0615798 & 3,1052465 \\
\hline $\log A_{3}$ & 5,0973650 & 6,4065347 & 4,5889514 & 5,8682055 \\
\hline $\log \boldsymbol{A}_{4}$ & 8,149924 & 9,655978 & 7,6208431 & 9,083199 \\
\hline $\log \boldsymbol{A}_{5}$ & 11,5834 & 13,2496 & 11,040582 & 12,653148 \\
\hline $\log A_{6}$ & 15,33 & 17,130 & 14,77604 & 16,51613 \\
\hline $\log A_{\bar{\tau}}$ & & 21,3 & 18,7780 & 20,6290 \\
\hline $\log A_{8}$ & & & 23,01 & 24,96 \\
\hline $\log A_{9}$ & & & & 29,48 \\
\hline
\end{tabular}

für $\theta=1$, also $\gamma=\frac{3}{2}$ (nach Wertheim):

\begin{tabular}{|c|c|c|c|c|}
\hline $\log A_{1}$ & 0,6812413 & 1,3521826 & 0,2242682 & 0,9393022 \\
\hline $\log A_{2}$ & 2,5563026 & 3,5940911 & 2,0189332 & 3,0664444 \\
\hline $\log \boldsymbol{A}_{3}$ & 5,1204304 & 6,4136351 & 4,5462236 & 5,827905 \\
\hline $\log \boldsymbol{A}_{4}$ & 8,174058 & 9,663768 & 7,5783037 & 9,042324 \\
\hline $\log \boldsymbol{A}_{5}$ & 11,6083 & 13,2578 & 10,998263 & 12,612037 \\
\hline $\log A_{6}$ & 15,35 & 17,138 & 14,73392 & 16,47493 \\
\hline $\log \boldsymbol{A}_{7}$ & & 21,3 & 18,736 & 20,5877 \\
\hline $\log A_{8}$ & & & 22,97 & 24,92 \\
\hline $\log \boldsymbol{A}_{9}$ & & & & 29,44 \\
\hline
\end{tabular}

Hieraus gehen folgende Werthe von

$$
\log \left(\lambda_{n \mu} l\right)^{4}
$$

hervor :

für $\theta=\frac{1}{2}$ :

\begin{tabular}{ccccc}
$\mu$ & $n=0$ & $n=1$ & $n=2$ & $n=3$ \\
0 & - & - & 0,27837 & 1,00651 \\
1 & 0,69367 & 1,41553 & 1,89117 & 2,24693 \\
2 & 1,96308 & 2,34829 \\
\multicolumn{4}{c}{ für $\theta=1:$} \\
0 & - & $-\quad$ & 0,23638 & 0,97014 \\
1 & 0,71168 & 1,42012 & 1,88997 & 2,24298 \\
2 & 1,96712 & 2,35022 &
\end{tabular}

In der folgenden Tafel sind die Tonverhältnisse, welche Chladni gefunden hat, zusammengestellt mit denen, welche die Rechnung giebt. Es sind die 
Töne angegeben, welche eine Scheibe geben kann, deren tiefster Ton $C$ ist. In den mit $\boldsymbol{C}$. überschriebenen Columnen finden sich die Töne, welche Chladni beobachtet hat, in den mit $\boldsymbol{P}$. überschriebenen die, welche die Rechnung unter der Voraussetzung $\theta=\frac{1}{2}$, in den mit $W$. überschriebenen die, welche die Rechnung unter der Voraussetzung $\theta=1$ geliefert hat. Die Angaben beziehen sich alle auf die gleichschwebende Temperatur ${ }^{*}$ ). Jeder berechnete Ton ist durch den ihm zunächst liegenden Ton der Scale bezeichnet, dem ein + oder - beigegfügt ist, je nachdem jener etwas höher oder tiefer als dieser war.

\begin{tabular}{|c|c|c|c|c|c|c|c|c|c|c|c|c|}
\hline$\mu$ & \multicolumn{3}{|c|}{$n=0$} & \multicolumn{3}{|c|}{$n=1$} & \multicolumn{3}{|c|}{$n=2$} & \multicolumn{3}{|c|}{$n=3$} \\
\hline & Ch. & $\boldsymbol{P}$. & $\boldsymbol{W}$. & Ch. & $\boldsymbol{P}$ & $\boldsymbol{W}$ & $C h$. & $\boldsymbol{P}$. & $\boldsymbol{W}$. & Ch. & $\boldsymbol{P}$. & $\boldsymbol{W}$. \\
\hline 0 & - & - & - & - & - & - & $C$ & $\boldsymbol{C}$ & $C$ & $d$ & dis - & dis - \\
\hline 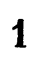 & Gis & Gis + & $\boldsymbol{A}+$ & $\boldsymbol{b}$ & $h-$ & $c-$ & $\bar{g}$ & $\overline{g i s}+$ & $\bar{a}-$ & $\overline{\overline{\boldsymbol{d}}} . \overline{\overline{d i s}}$ & $\overline{\overline{\operatorname{dis}}}+$ & $\overline{\bar{e}}-$ \\
\hline & $i s+$ & $\bar{b}-$ & $\bar{b}+$ & & $\overline{\bar{f}}+$ & $\overline{\overline{f i s}}$ & & & & & & \\
\hline
\end{tabular}

Es zeigen sich hier nicht unerhebliche Abweichungen der beobachteten Töne von den durch die beiden Rechnungen ermittelten. Die beobachteten Töne stimmen mit den aus der Poisson'schen Annahme $\left(\theta=\frac{1}{2}\right)$ ermittelten etwas besser überein, als mit den aus der Wertheim'schen Annahme $(\theta=1)$ berechneten; doch ist die Abweichung bei jenen zu grofs, als dafs hieraus ein Schlufs gegen diese Annahme gezogen werden könnte.

Ich wende mich jetzt zur Vergleichung einiger numerischen Resultate, welche die Theorie in Bezug auf die Knotenlinien giebt, mit den entsprechenden Resultaten der Beobachtung. Hr. Professor Srehlke hat die Güte gehabt, mir die Ergebnisse einiger Messungen von ausgezeichneter Genauigkeit mitzutheilen, die er an zwei kreisförmigen Glasscheiben angestellt hat. Diese Scheiben waren mit derselben Sorgfalt gearbeitet, wie die quadratischen Scheiben, an denen er die Messungen angestellt hat, die von ihm in Dove's Repertorium Bd. III. S. 113 bekannt gemacht sind; die eine von ihnen hatte ungefähr 6 Zoll Durchmesser und 1 Linie Dicke, die andere 7 Zoll Durchmesser und 1,1 Linie Dicke. Zum Beweise der Vollkommenheit der Scheiben und der Genauigkeit der Messungsmethode kann die Kleinheit der Unterschiede der folgenden Zahlen dienen, die durch Messung verschiedener Durchmesser des Knotenkreises ohne Knotendurchmesser auf einer Scheibe gefunden wurden.

*) Chladni sagt zwar nicht ausdrücklich in seiner Akustik, aus welcher seine Angaben genommen sind, dafs dieselben sich auf die gleichschwebende Temperatur beziehen; doch scheint es unzweifelhaft, dafs dem so ist. 
4. Kirchhoff, ̈̈b. d. Gleichgewicht u.d. Bewegung einer elastischen Scheibe. 87

\begin{tabular}{cc} 
Erste Seite. & Kehrseite derselben Scheibe. \\
$24^{\mathrm{L}}, 415$ & $24^{\mathrm{L}}, 42$ \\
43 & 44 \\
44 & 425 \\
425 & 43 \\
405 & 415 \\
\cline { 2 - 2 } Mittel $24^{\mathrm{L}}, 423$ & $24^{\mathrm{L}}, 426$
\end{tabular}

Eben so regelmäfsig als diese Scheibe, welche mit I bezeichnet werden mag, zeigte sich die andere 7zöllige, welche II genannt werden soll. Ich will die Werthe der Radien der Knotenkreise, welche diese beiden Scheiben ergeben haben, zusammenstellen mit den Werthen, welche die Rechnung bei der Poisson'schen oder der Wertheim'schen Annahme von $\theta$ giebt.

$$
\begin{array}{l|l|c|c|c} 
& \multicolumn{2}{|c|}{\text { Beobachtung }} & \multicolumn{2}{|c}{\text { Rechnung }} \\
\hline \text { I } & \text { II } & \text { P. } & W . \\
n=0, \mu=1 & l .0,6792 & l .0,6782 & l .0,68062 & l .0,67941 \\
n=1, \quad \mu=1 & l .0,7811 & l .0,7802 & l .0,78136 & l .0,78088
\end{array}
$$

Den Radius des Knotenkreises, der dem Tone $(n=0, \mu=1)$ entspricht, hat auch Savart gemessen; er fand bei drei verschiedenen Scheiben die folgenden Werthe:

$$
l .0,6819, \quad l .0,6798, \quad l .0,6812 \text {. }
$$

Diese Angaben theilt Poisson bei Gelegenheit seiner Untersuchungen über die Schwingungen einer kreisförmigen Platte in der oben citirten Abhandlung mit. Poisson hat dort für den Fall $n=0$ und unter der Annahme $\theta=\frac{1}{2}$ die tiefsten Töne und die zu diesen gehörigen Knotenkreise berechnet.

Die aus der Wertheim'schen Annahme abgeleiteten Resultate weichen von den aus der Poisson'schen abgeleiteten nur wenig ab; mit den Strehlkeschen Beobachtungen stimmen jene noch besser überein, als diese. Wie mir scheint, spricht dieses aber nicht gegén die Poisson'sche Annahme, denn eine vollkommene Übereinstimmung zwischen der Theorie und dem Versuche darf man nicht erwarten, weil die dem Versuche unterworfenen Scheiben nicht die Eigenschaften in aller Strenge besitzen, welche in der Theorie ihnen beigelegt werden.

Hr. Strehlke hat, aufser den angeführten, mir noch die Resultate einiger anderen Messungen mitgetheilt, die von ihm an weniger vollkommenen Scheiben angestellt sind. Ich lasse dieselben folgen, zugleich mit den entsprechen- 
88 4. Kirchhoff, üb. d. Gleichgewicht u. d Bcwegung einer elastischen Scheibe.

den Zahlen, welche die Rechnung unter der Annahme $\theta=\frac{1}{2}$ und unter der Annahme $\theta=1$ gegeben hat.

Radien der Knotenkreise.

\begin{tabular}{l|llll|l|l}
\hline & \multicolumn{3}{|c|}{ Beobachtung } & \multicolumn{2}{c}{ Rechnung } \\
& & & & $\theta=\frac{1}{2}$ & $\theta=1$ \\
\hline$n=1, \mu=1$ & 0,781 & 0,783 & 0,781 & 0,783 & 0,78136 & 0,78088 \\
$n=2, \mu=1$ & 0,79 & 0,81 & 0,82 & & 0,82194 & 0,82274 \\
$n=3, \mu=1$ & 0,838 & 0,842 & & & 0,84523 & 0,84681 \\
$n=1, \mu=2$ & 0,488 & 0,492 & & & 0,49774 & 0,49715 \\
0,869 & 0,869 & & & 0,87057 & 0,87015
\end{tabular}

Der Radius der Scheibe ist hierbei $=1$ gesetzt.

Im Januar 1850. 